\title{
Exploring the Connection between Parsec-scale Jet Activity and Broadband Outbursts in 3C 279
}

\author{
B. Rani ${ }^{1,13}$, S. G. Jorstad ${ }^{2,3}$ (1) , A. P. Marscher ${ }^{2}$ (10) I. Agudo ${ }^{4}$, K. V. Sokolovsky ${ }^{5,6,7}$, V. M. Larionov ${ }^{3,8}$ (1), P. Smith ${ }^{9}$, \\ D. A. Mosunova ${ }^{6}$, G. A. Borman ${ }^{10}$, T. S. Grishina ${ }^{3}$, E. N. Kopatskaya ${ }^{3}$, A. A. Mokrushina ${ }^{3,8}$, D. A. Morozova ${ }^{3}$, S. S. Savchenko ${ }^{3}$, \\ Yu. V. Troitskaya ${ }^{3}$, I. S. Troitsky ${ }^{3}$, C. Thum ${ }^{11}$, S. N. Molina ${ }^{4}$, and C. Casadio ${ }^{4,12}$ \\ ${ }^{1}$ NASA Goddard Space Flight Center, Greenbelt, MD 20771, USA; bindu.rani@nasa.gov \\ 2 Institute for Astrophysical Research, Boston University, 725 Commonwealth Avenue, Boston, MA 02215, USA \\ ${ }^{3}$ Astron. Inst., St.-Petersburg State Univ., Russia \\ ${ }^{4}$ Instituto de Astrofísica de Andalucía (CSIC), Apartado 3004, E-18080 Granada, Spain \\ 5 IAASARS, National Observatory of Athens, Vas. Pavlou \& I. Metaxa, GR-15 236 Penteli, Greece \\ ${ }^{6}$ Astro Space Center of Lebedev Physical Institute, Profsoyuznaya St. 84/32, 117997 Moscow, Russia \\ 7 Sternberg Astronomical Institute, Moscow State University, Universitetskii pr. 13, 119992 Moscow, Russia \\ ${ }^{8}$ Pulkovo Observatory, St.-Petersburg, Russia \\ ${ }^{9}$ Steward Observatory, University of Arizona, Tucson, AZ 85721, USA \\ ${ }^{10}$ Crimean Astrophysical Observatory, P/O Nauchny, Crimea, ${ }^{\dagger} 298409$, Russia \\ ${ }^{11}$ Instituto de Radio Astronomía Millimétrica, Avenida Divina Pastora, 7, Local 20, E-18012 Granada, Spain \\ 12 Max-Planck-Institut für Radioastronomie, Auf dem Hügel, 69, D-53121, Bonn, Germany \\ Received 2017 November 5; revised 2018 March 14; accepted 2018 March 15; published 2018 May 9
}

\begin{abstract}
We use a combination of high-resolution very long baseline interferometry (VLBI) radio and multiwavelength flux density and polarization observations to constrain the physics of the dissipation mechanism powering the broadband flares in 3C 279 during an episode of extreme flaring activity in 2013-2014. Six bright flares superimposed on a long-term outburst are detected at $\gamma$-ray energies. Four of the flares have optical and radio counterparts. The two modes of flaring activity (faster flares sitting on top of a long-term outburst) present at radio, optical, and $\gamma$-ray frequencies are missing in X-rays. X-ray counterparts are only observed for two flares. The first three flares are accompanied by ejection of a new VLBI component (NC2), suggesting the $43 \mathrm{GHz}$ VLBI core as the site of energy dissipation. Another new component, NC3, is ejected after the last three flares, which suggests that the emission is produced upstream from the core (closer to the black hole). The study therefore indicates multiple sites of energy dissipation in the source. An anticorrelation is detected between the optical percentage polarization (PP) and optical $/ \gamma$-ray flux variations, while the PP has a positive correlation with optical $/ \gamma$-ray spectral indices. Given that the mean polarization is inversely proportional to the number of cells in the emission region, the PP versus optical $/ \gamma$-ray anticorrelation could be due to more active cells during the outburst than at other times. In addition to the turbulent component, our analysis suggests the presence of a combined turbulent and ordered magnetic field, with the ordered component transverse to the jet axis.
\end{abstract}

Key words: galaxies: active - quasars: individual (3C 279) - radio continuum: galaxies - galaxies: jets - gamma rays: galaxies

\section{Introduction}

Combined information from the high-resolution images obtained using very long baseline interferometry (VLBI) radio and broadband intensity variations has been proven to be one of the most useful approaches to locate and probe the high-energy radiation processes in blazars-active galactic nuclei (AGNs) in which one of the jets points close to our line of sight. Investigation of intensity variations in individual sources in combination with the VLBI component ejection/kinematics indicates that high-energy emission is associated with the compact regions in relativistic jets (e.g., Marscher 2008; Jorstad et al. 2010; Agudo et al. 2011; Rani et al. 2014, 2015, 2016, and references therein). A recent study of blazar S5 $0716+714$ by Rani et al. (2014) reported a significant correlation between the $\gamma$-ray flux and the direction of the jet

\footnotetext{
13 NPP Fellow.

$\dagger$ While the AAS journals adhere to and respect UN resolutions regarding the designations of territories (available at http://www.un.org/press/en), it is our policy to use the affiliations provided by our authors on published articles.
}

outflow variations. This study implies a causal connection between the jet morphology and the observed $\gamma$-ray flares.

We present here an investigation of parsec-scale jet morphology evolution in the blazar 3C 279 during an episode of extreme flaring activity at $\mathrm{GeV}$ energies in 2014, using $43 \mathrm{GHz}$ VLBI images. The focus of this study is to explore the total intensity and polarization properties and their connection with the broadband flux variations observed during this bright $\gamma$-ray activity phase of the source.

The flat-spectrum radio quasar (FSRQ) 3C 279, at a redshift of $z=0.538$ (Burbidge \& Rosenberg 1965), has a black hole mass in the range of $(3-8) \times 10^{8} M_{\odot}$ (Gu et al. 2001 ; Woo \& Urry 2002). It has a bright jet extending up to kiloparsec scales (Cheung 2002) and exhibits a range of apparent velocities from 4 to $20 c$ (Lister et al. 2013). The parsec-scale jet is aligned close to the observer's line of sight $\left(\leqslant 2^{\circ}\right.$; Jorstad et al. 2004; Lister et al. 2013). Because of its strong optical polarization and flux variability, 3C 279 is often classified as an optically violently variable quasar. Polarimetric observations have detected both linearly and circularly polarized emission from the parsec-scale jet of 3C 279 (Wardle et al. 1998; Taylor 2000; 
Homan et al. 2009). Moreover, the observed radiation at optical frequencies is also highly polarized in the source, e.g., an observed value of $45.5 \%$ in the optical $U$ band (Mead et al. 1990). Wagner \& Mannheim (2001) detected variable optical circular polarization in 3C 279 exceeding $1 \%$.

With its high flux density and prominent variations in total intensity and polarization, 3C 279 has been the subject of several intensive multiwavelength campaigns (e.g., Maraschi et al. 1994; Hartman et al. 1996; Wehrle et al. 1998; Böttcher et al. 2007; Chatterjee et al. 2008; Larionov et al. 2008; Collmar et al. 2010; Hayashida et al. 2012, 2015; Kang et al. 2015). In particular, prominent $\gamma$-ray flares were recorded in the source by the Energetic Gamma-Ray Experiment Telescope (EGRET) on board the Compton Gamma-Ray Observatory (CGRO; Hartman et al. 1992), and the Fermi Large Area Telescope (LAT) has detected the source regularly since its launch in 2008 (Abdo et al. 2010). In 2006, the Major Atmospheric Gamma-Ray Imaging Cherenkov (MAGIC) telescope observed $\mathrm{TeV}(E>200 \mathrm{GeV})$ emission from the source (Albert et al. 2008). In 2013 December, the source went through a series of extremely rapid $\gamma$-ray flares, reaching a flux level $F(E>100 \mathrm{MeV})$ of $10^{-5}$ photons $\mathrm{cm}^{-2} \mathrm{~s}^{-1}$ on timescales of the order of a few hr (Hayashida et al. 2015). In 2015 June, record-breaking flaring activity was observed (Cutini 2015) with the highest measured flux $F(E>100 \mathrm{MeV})$ of $3.6 \times 10^{-5}$ photons $\mathrm{cm}^{-2} \mathrm{~s}^{-1}$ (Ackermann et al. 2016).

This paper is structured as follows. Section 2 provides a brief description of the observations and data reduction. In Section 3, we report and discuss our results. Finally, a summary and conclusions are given in Section 4. Throughout this paper, we adopted a $\Lambda$ CDM cosmology with $\Omega_{m}=0.27, \Omega_{\lambda}=0.73$, and $H_{0}=71 \mathrm{~km} \mathrm{~s}^{-1} \mathrm{Mpc}^{-1}$ (Spergel et al. 2003). At a redshift of 0.538 , the luminosity distance $d_{L}$ is $3085 \mathrm{Mpc}$, and an angular separation of 1 mas translates into a linear distance of $6.32 \mathrm{pc}$.

\section{Multifrequency Data: Observations and Data Reduction}

From 2013 November 01 to 2014 August 30, the broadband flaring activity of the FSRQ 3C 279 was extensively covered using both ground- and space-based observing facilities. The following subsections summarize the observations and data reduction.

\subsection{VLBA Observations}

The $7 \mathrm{~mm}$ VLBA observations of the source were obtained in the course of a program ${ }^{14}$ of monthly monitoring of bright $\gamma$-ray blazars at $43 \mathrm{GHz}$. The details of the observations can be found in Jorstad et al. (2017). The data reduction is performed using the Astronomical Image Processing System (AIPS) and Difmap (Shepherd 1994). Details of the data reduction are described in Jorstad et al. (2005, 2017). We model-fit the brightness distribution of the source using circular Gaussian components using Difmap, and the model-fit parameters (peak flux density, distance from $(0,0)$ coordinates, position angle, and size) are listed in Table 2 in the appendices. The polarization parameters (polarized flux density and electric vector position angle (EVPA)) are determined by model-fitting the stokes $Q$ and $U$ in $(u, v)$ data. The polarized flux density is given by $\sqrt{Q_{\text {flux }}^{2}+U_{\text {flux }}^{2}}$ and the EVPA by $0.5 \times \arctan \left(U_{\text {flux }} / Q_{\text {flux }}\right)$.

\footnotetext{
${ }^{14}$ VLBA-BU-BLAZARS, http://www.bu.edu/blazars.
}

\subsection{Radio Observations}

The 230 and $345 \mathrm{GHz}$ data are provided by the Submillimeter Array (SMA) Observer Center ${ }^{15}$ database (Gurwell et al. 2007). The $230 \mathrm{GHz}$ total flux density data are complemented by the data from the Polarimetric Monitoring of Active Galactic Nuclei at Millimetre Wavelengths (POLAMI) program ${ }^{16}$ (Agudo et al. 2018a, 2018b; Thum et al. 2018). POLAMI is a long-term program to monitor the polarimetric properties (Stokes $I, Q, U$, and $V$ ) of a sample of around 40 bright AGNs at 3.5 and $1.3 \mathrm{~mm}$ wavelengths with the IRAM $30 \mathrm{~m}$ telescope near Granada, Spain. The program has been running since 2006 October, and it currently has a time sampling of $\sim 2$ weeks. The XPOL polarimetric observing setup has been routinely used as described in Thum et al. (2008) since the start of the program. The reduction and calibration of the POLAMI data presented here are described in detail in Agudo et al. (2010, 2014, 2018a). We also make use of the $15 \mathrm{GHz}$ Owens Valley Radio Observatory (OVRO) data publicly available at the OVRO website. ${ }^{17}$ Details of the observations and data reduction are described in Richards et al. (2011).

\subsection{Optical Observations}

During our campaign period, the source was simultaneously observed in six optical/UV filters $(U, B, V, W 1, W 2$, and $M 2)$ by Swift/UVOT (Roming et al. 2005). We used the uvotsource tool from HEASoft v6.16 for the data analysis (Poole et al. 2008). A step-by-step data reduction procedure is described in Rani et al. (2017), to which we refer for details. We have also included optical/IR data at the $B, V, R, J$, and $K$ passbands from the Small and Moderate Aperture Research Telescope System (SMARTS5) monitoring program. The reduction and analysis of this data are described in Bonning et al. (2012).

We also make use of the optical $R$-passband flux and polarization observations provided by the Steward Observatory blazar monitoring program ${ }^{18}$; the details of the observations and data reduction are described in Smith (2016). Additional optical $R$-band polarimetric observations were obtained at the $1.83 \mathrm{~m}$ Perkins telescope of the Lowell Observatory (Flagstaff, AZ) using the PRISM camera. ${ }^{19}$ The details of the observations and data reduction are given in Jorstad \& Marscher (2016). We also used polarimetric and photometric data from the AZT-8 (Crimean Astrophysical Observatory) and LX-200 (St. Petersburg, Russia) telescopes. The details of the observations and data reduction are given in Larionov et al. (2008).

Finally, the data were corrected for the Galactic extinction using the coefficients of Schlegel et al. (1998) for different filters. ${ }^{20} \mathrm{We}$ converted the magnitudes to fluxes using the central wavelengths for each filter as calibrated by Poole et al. (2008) for the space-based observations. For the ground-based observations, the zero-point fluxes ${ }^{21}$ corresponding to the observed wavelengths were used for magnitude-to-flux conversion.

\footnotetext{
15 http://sma1.sma.hawaii.edu/callist/callist.html

16 https://polami.iaa.es

${ }^{17}$ http://www.astro.caltech.edu/ovroblazars/

$18 \mathrm{http}: / /$ james.as.arizona.edu/ psmith/Fermi/

19 http://www.bu.edu/prism/

${ }^{20}$ Search for 3C 279 at http://ned.ipac.caltech.edu/.

21 http://ssc.spitzer.caltech.edu/warmmission/propkit/pet/magtojy/ref.html
} 


\subsection{X-Ray Observations}

$\mathrm{X}$-ray observations $(0.3-10 \mathrm{keV})$ of the source were obtained using the Swift/XRT telescope (Burrows et al. 2005) in both the photon-counting and windowed-timing mode. The data are analyzed using the standard xrtpipeline v0.13.2. The spectra in individual time bins were modeled with XSPEC v12.9.0 to extract the photon counts and indices. We used the absorbed power law with the total $\mathrm{HI}$ column density fixed to the Galactic value $\left(N_{\mathrm{H}}=0.212 \times 10^{21}\right.$; Kalberla et al. 2005) to model the $0.3-10 \mathrm{keV}$ spectrum, which provided an acceptable fit at all epochs. The details of the observations and data reduction are discussed in Rani et al. (2017).

\subsection{Gamma Rays}

Here we employed the $100 \mathrm{MeV}-300 \mathrm{GeV}$ data of the source observed in survey mode by the Fermi/LAT (Atwood et al. 2009). The LAT data were analyzed using the standard ScienceTools (software version v10.r0.p5) and instrument response function P8R2_SOURCE_V6. Photons in the Source event class were selected for the analysis. We analyzed a region of interest (ROI) of $20^{\circ}$ centered at the position of 3C 279 using a maximum-likelihood algorithm (Mattox et al. 1996). For the unbinned likelihood analysis, ${ }^{22}$ all 3FGL (Acero et al. 2015) sources within the ROI were included. We also included the Galactic diffuse background (gll_iem_v06.fits) and isotropic background (iso_P8R2_SOURCE_V6_v06.txt) emission components. ${ }^{23}$ Model parameters for sources within $5^{\circ}$ of the center of the ROI were kept free. Model parameters for variable sources (variability index $\geqslant 72.44$ in the 3 FGL catalog) were also set free, while the model parameters of nonvariable sources beyond $5^{\circ}$ were fixed to their catalog values.

To characterize the variability properties of the source, we computed the photon-flux light curve of the source at $E$ above $E_{0}$, where $E_{0}$ is the decorrelation energy (Lott et al. 2012), which minimizes the correlations between integrated photon flux and photon index. During our campaign, we obtained $E_{0}=219 \mathrm{MeV}$. We generated the constant uncertainty (15\%) light curve above $E_{0}$ through the adaptive-binning method following Lott et al. (2012). The bin size ranges between 0.07 and 31.06 days with an average of 5.43 days. The adaptive binned light curve is produced by modeling the spectra by a simple power law, $N(E)=N_{0} E^{-\Gamma}, N_{0}$ : prefactor, and $\Gamma$ : power-law index.

\section{Results and Discussion}

This section presents the light-curve analysis-flux and polarization variability at different energy bands and the evolution of the jet brightness. We also investigate the connection between jet kinematics and broadband flaring activity.

\subsection{Observed Outburst and Its Multifrequency Behavior}

The observed broadband flux density variations in 3C 279 during a major $\gamma$-ray outburst are plotted in Figure 1. The source became active at $\mathrm{GeV}$ energies in early 2013 November, and the flaring activity continued until 2014 August. Hereafter, we will use $\mathrm{JD}^{\prime}$ (defined as $\mathrm{JD}-2,455,000$ ) for time. In

\footnotetext{
$\overline{22}$ http://fermi.gsfc.nasa.gov/ssc/data/analysis/scitools/likelihood_ tutorial.html

${ }^{23}$ https://fermi.gsfc.nasa.gov/ssc/data/access/lat/BackgroundModels.html
}

Figure 1, the cyan dashed lines labeled "A" and "D" mark the beginning and end of the outburst, respectively. The source displayed multiple flares across the whole electromagnetic spectrum over this period, which we visually inspected and labeled as follows. The blue dotted-dashed lines (1-6) are marked close to the peak of the rapid $\gamma$-ray flares labeling the broadband flares "G" for $\gamma$-rays, "X" for X-rays, "O" for optical, and "R" for radio, followed by the number. For instance, the $\gamma$-ray flares should be read as G1-G6.

Flare 2 is observed close in time at $\gamma$-ray and X-ray energies. The first two flares are accompanied by an increase in the source brightness at radio frequencies. At optical frequencies (panel (c)), the source is still in the quiescent phase. The peak of flare G3 coincides with a minimum in the X-ray light curve but has optical and radio counterparts and a delayed response in $\mathrm{X}$-rays. In comparison to $\mathrm{X} 1$ and $\mathrm{X} 2, \mathrm{X} 3$ has a rather low magnitude. Because of the gap in observations at X-ray energies, it is hard to determine the absence/presence of an $\mathrm{X}$-ray counterpart for flare G4, but the flare has a nearsimultaneous optical and radio counterpart. A prominent optical flare (labeled "3.5") is seen between flares 3 and 4. The flare $\mathrm{O} 3.5$ is accompanied by similar activity at the radio $(230 \mathrm{GHz})$ band; however, the observed variations are quite mild at X-ray and $\gamma$-ray energies. Flare 5 is the only one that is observed quasi-simultaneously at all energies (radio to $\gamma$-rays). Flare 5 has a double feature in $\gamma$-rays and X-rays (see Figures 2 and 4).

The other two interesting states in the broadband activity are marked "B" and "C." After the first five $\gamma$-ray flares, a local minimum (B) was observed simultaneously at all frequencies. The local minimum was followed by a concurrent flare (flare 6) at radio, optical, and $\gamma$-ray energies; no significant activity was observed in the X-ray regime during this period. Period $\mathrm{C}$ marks the end of flare 6 , which happened to be almost simultaneous for all light curves. A continuous decay in the source brightness was observed between periods C and D, which we refer to as the post-outburst plateau.

\subsubsection{Gamma-Ray Variability}

Figure 2 displays details of the observed $\gamma$-ray flaring activity of 3C 279. The rapid flares (labeled 1-6) were superimposed on top of a broad outburst. The source started to brighten from a flux level $F(E>219 \mathrm{MeV}) \sim 3.3 \times 10^{-7}$ photons $\mathrm{cm}^{-2} \mathrm{~s}^{-1}$ on $\mathrm{JD}^{\prime} \sim 1610$ and returned to the same brightness level on $\mathrm{JD}^{\prime} \sim 1850$. The green lines in Figure 2 mark the observed brightness level $\left(\sim 3.3 \times 10^{-7}\right.$ photons $\left.\mathrm{cm}^{-2} \mathrm{~s}^{-1}\right)$ of the source before and after the outburst period. Significant variations in $\gamma$-ray photon index were observed during this period. The estimated $\Gamma$ value is 2.7 at the beginning and end of the outburst. A significant spectral variation is observed for the individual flares (1-6). For each flare, the spectrum gets harder at the peak, and later it softens again. The fastest observed $\gamma$-ray flare during this activity period had a flux-doubling timescale of $\sim 2 \mathrm{hr}$ and a very hard $\gamma$-ray spectrum with $\Gamma=1.7$ (Hayashida et al. 2015).

In Figure 3, we plot the $\gamma$-ray photon flux values as a function of photon index. A clear spectral hardening with an increase in source brightness can be seen here. Since we investigate the correlation above the decorrelation energy $\left(E_{0}\right)$, the estimated correlation is not sensitive to the LAT instrumental bias. A Pearson correlation analysis reveals a significant correlation between the two. Formally, we obtained 


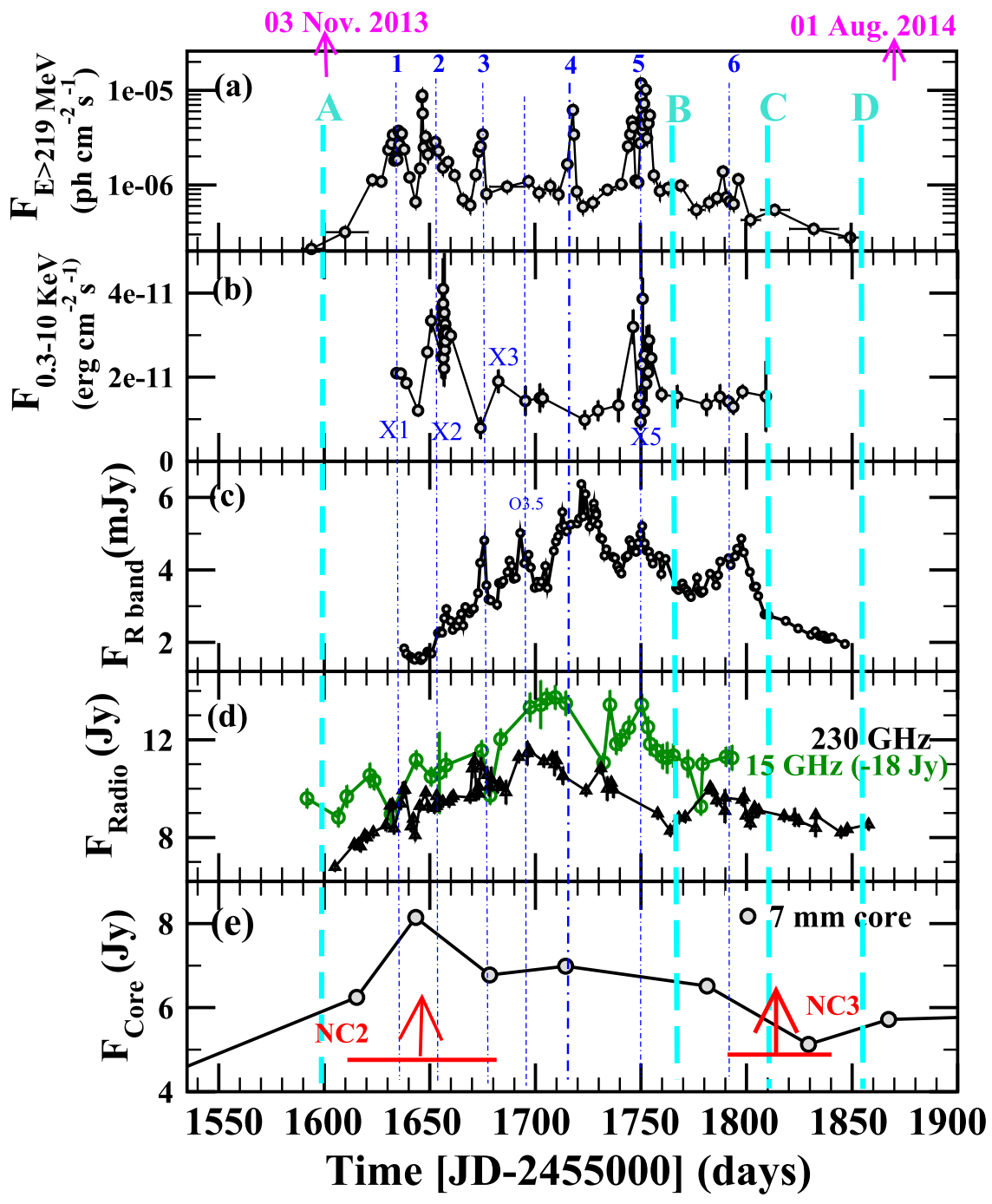

Figure 1. Broadband flux light curves of 3C 279: (a) $\gamma$-ray light curve at $E>219 \mathrm{MeV}$, (b) X-ray light curve at $0.3-10 \mathrm{keV}$, (c) optical $R$-passband light curve, (d) 230 and $15 \mathrm{GHz}$ radio flux density light curves, and (e) flux density variations in the $7 \mathrm{~mm}$ VLBI core. The red arrows mark the ejection times of new components (see Section 3.3 for details). As for other figures hereafter, the cyan dashed lines (labeled A-D) represent the different flaring activity states of the source (see Section 3.1 for more details). The peaks of the rapid $\gamma$-ray flares (1-6) are marked with blue dotted-dashed lines. The dashed line (labeled as 03.5) shows the time of an optical flare that has no obvious counterparts at other wavelengths. In all cases, the lines connecting the data points are simply to guide the eye.

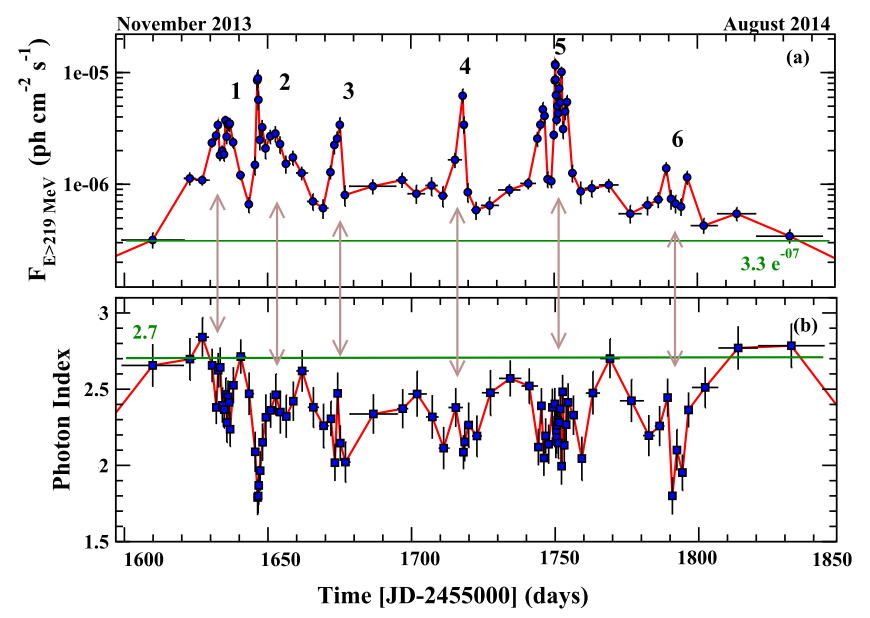

Figure 2. Gamma-ray photon flux (top) and photon index (bottom) light curves of $3 \mathrm{C} 279$. The bright $\gamma$-ray flare and the corresponding spectral variations are shown with the gray arrows. The photon flux and photon index values at the beginning and end of the outburst are marked with green lines.

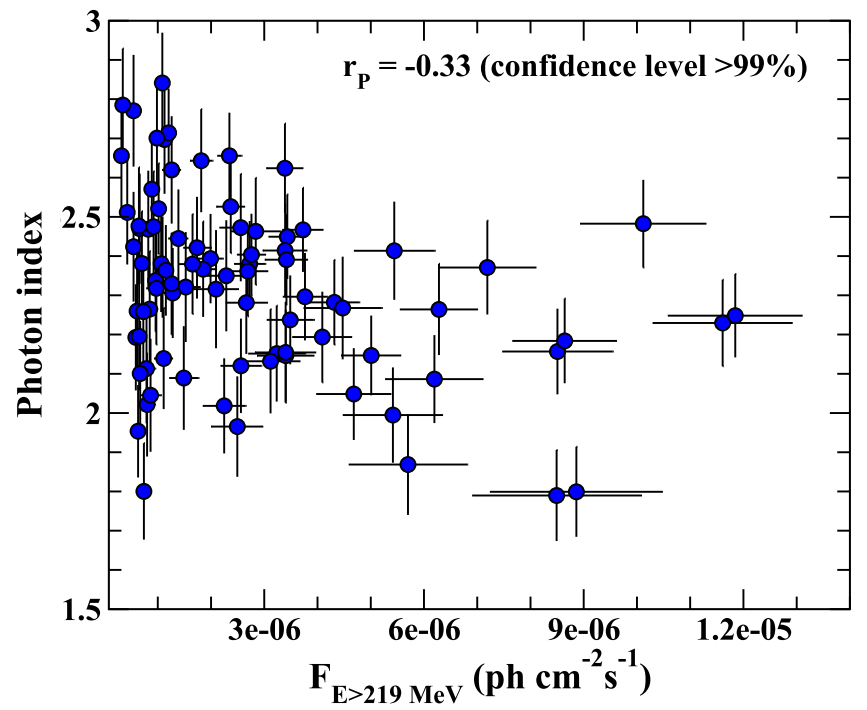

Figure 3. Gamma-ray photon index vs. photon flux for $E>219 \mathrm{MeV}$. 


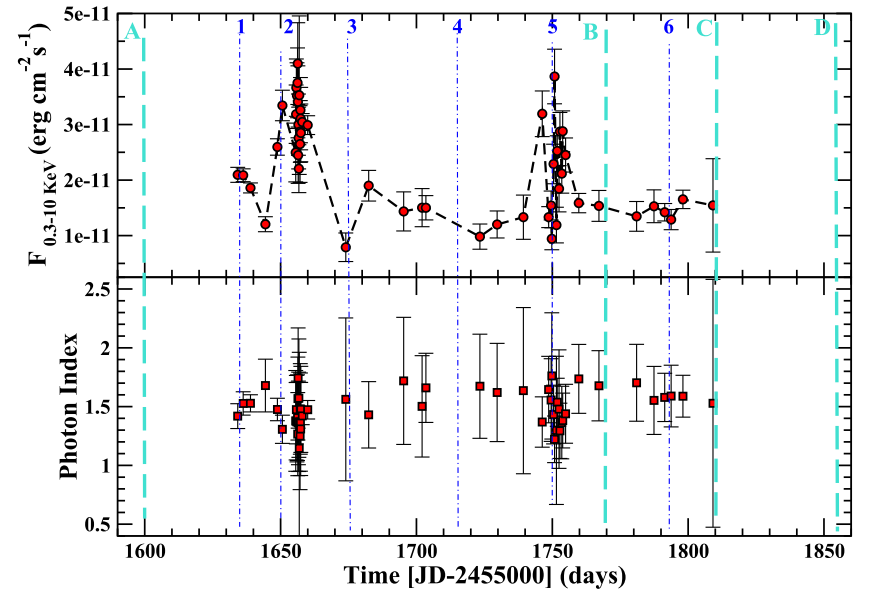

Figure 4. Energy flux (top) and photon index (bottom) light curves of 3C 279 at the $0.3-10 \mathrm{KeV} \mathrm{X}$-ray bands (vertical dotted-dashed and dashed lines are the same as in Figure 1).

$r_{\mathrm{P}}{ }^{24}=-0.33$ and a $p$-value $=0.0019$, which suggests a significant anticorrelation at a confidence level of $>99 \%$. It is therefore evident that not only the major outburst but also the individual $\gamma$-ray flares are characterized by significant spectral hardening, although with substantial scatter.

\subsubsection{X-Ray Variability}

The X-ray energy flux and photon index light curves are shown in Figure 4. Compared to the $\gamma$-ray light curve, the $\mathrm{X}$-ray observations were rather sparse. We could identify two prominent flares in the X-ray light curve. Apparently, the first X-ray flare coincides with flare G2 in $\gamma$-rays, and the second X-ray flare coincides with flare G5. While the X-ray energy flux is varying by a factor of more than four, the photon index remains consistent with the mean value of $\Gamma_{X}=1.47 \pm 0.01$.

\subsubsection{Variability at Optical Frequencies}

The observed near-IR, optical, and UV light curves of the source are shown in Figure 5. Similar to $\gamma$-rays, there are two modes of flaring activity at optical frequencies. Fast flares (timescale $\sim 20-30$ days) are superimposed on the broad outburst. No optical observations are available before $\mathrm{JD}^{\prime}=1635$ (2013 December). The flaring behavior is very similar for the near-IR, optical, and UV light curves.

The average IR-optical-UV (hereafter IOU) spectrum over the entire period of our observations is shown in Figure 6 (top). The observed IOU spectrum is steep until an upturn at UV frequencies. The excess emission could be due to thermal dominance either from the accretion disk or a broad-line region. The observed IOU spectrum can be well described by a power law, $F(\nu) \propto \nu^{-\alpha}$, where $\alpha$ is the spectral index. For the average spectrum, we obtained $\alpha=1.91 \pm 0.08$.

To investigate the spectral variations during the broad outburst, we constructed the IOU spectrum every 20 days. The spectral index is estimated via fitting the spectrum by a power law as described above. Prominent variations in the IOU spectral indices can be seen in Figure 6 (bottom). The IOU spectrum is steeper at the beginning of the outburst $\left(\alpha_{\mathrm{IOU}} \sim 2\right.$,

\footnotetext{
${ }^{24}$ Here $r_{\mathrm{P}}$ is the linear Pearson correlation coefficient and the $p$-value is the probability of the null hypothesis.
}

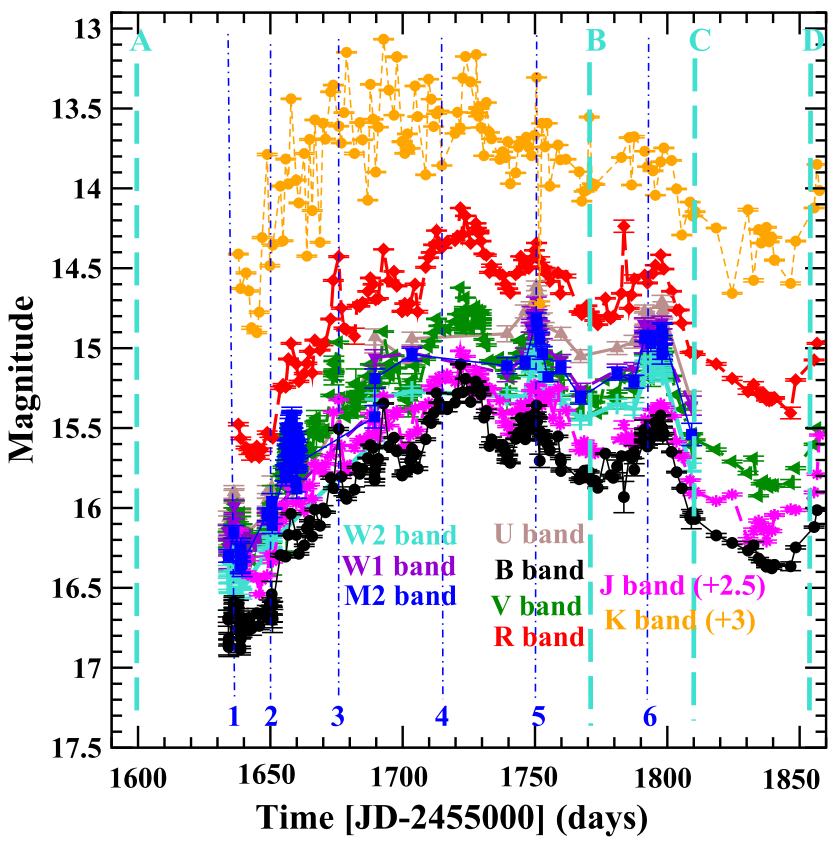

Figure 5. Near-IR ( $J$ and $K)$, UV ( $W 1, W 2$, and $M 2)$, and optical $(U, B, V$, and $R$ ) light curves of 3C 279 (vertical dotted-dashed and dashed lines are the same as in Figure 1).
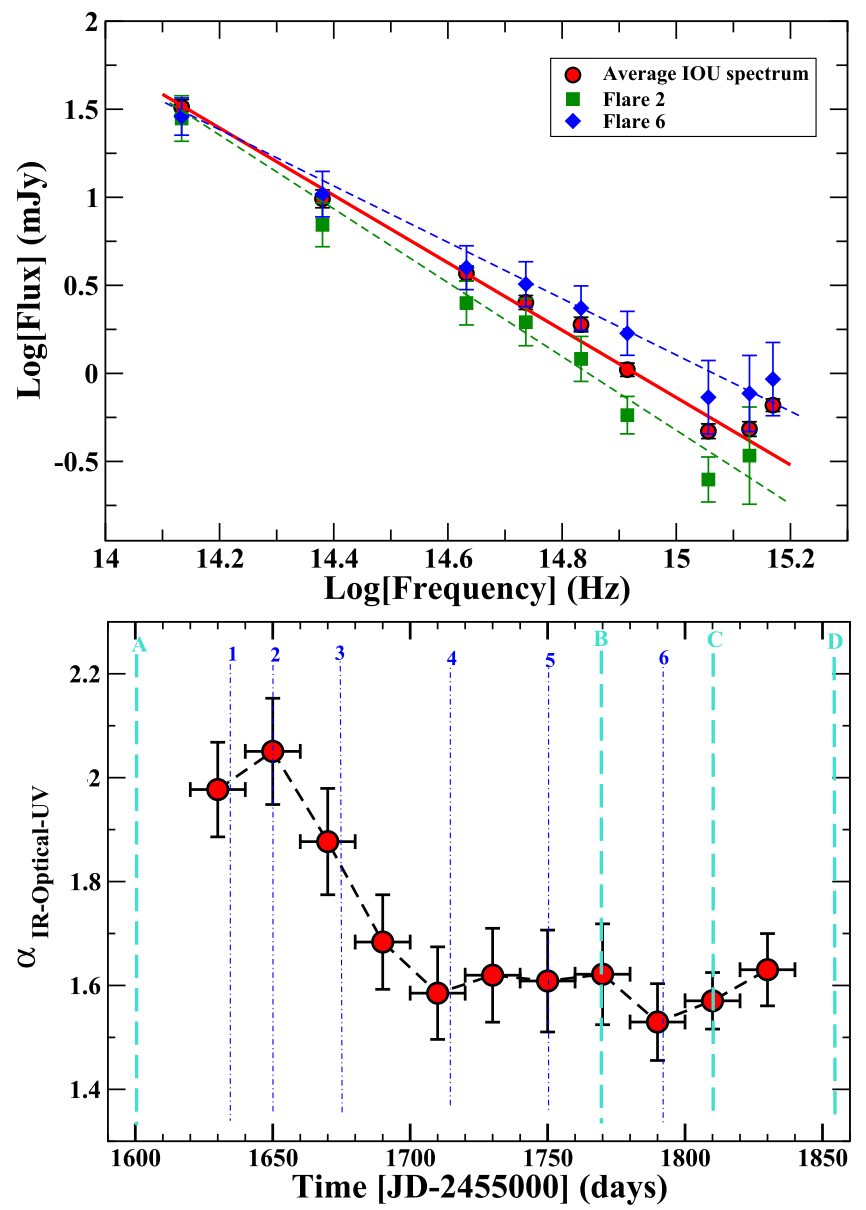

Figure 6. Top: average IOU spectrum (data points are the mean values, and the error bars represent their standard deviation) of 3C 279 over the entire period of observations and a change in spectral index from $\sim 2$ (Flare 2 in green) to $\sim 1.6$ (Flare 6 in blue). Bottom: spectral index variations (vertical dotted-dashed and dashed lines are the same as in Figure 1). 


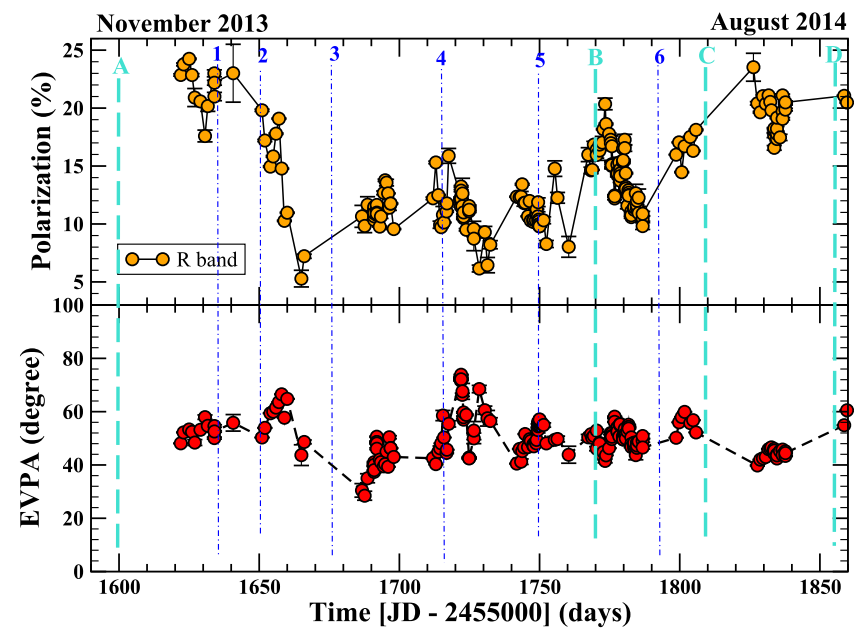

Figure 7. Optical $R$-band PP and EVPA variations (vertical dotted-dashed and dashed lines are the same as in Figure 1).

Flare 2), and the spectrum gets harder as the source brightness increases. The estimated $\alpha_{\text {IOU }}$ is $\sim 1.6$ (Flare 6) at the peak of the outburst $\left(\mathrm{JD}^{\prime} \sim 1720\right)$. However, the spectral variations are quite marginal during the decay phase of the outburst, with $\alpha_{\text {IOU }}$ remaining nearly consistent with a value of $\sim 1.6$.

Figure 7 presents the optical polarization variations observed in the source. The fractional polarization drops from $\sim 25 \%$ to $\sim 5 \%$ within the first 50 days of our observations; this period coincides with the beginning of the $\gamma$-ray outburst. The sharp drop in percentage polarization (PP) was followed by a slow rise (superimposed with rapid variations) until 2014 August. At the end of the outburst in 2014 August, the PP returned to its original value of $\sim 25 \%$. The optical polarization angle (EVPA, $\chi$ ) variations, on the other hand, are quite modest $\left(\Delta \chi \sim 60^{\circ}\right)$. Throughout the campaign period, the EVPA remained consistent with an average value of $\sim 50^{\circ}$, which is along the parsec-scale jet direction of $\sim-135^{\circ}$ (Jorstad et al. 2017).

\subsubsection{Variability at Radio Frequencies}

In Figure 8, we plot the radio flux density light curves sampled at the $15,86,230$, and $350 \mathrm{GHz}$ bands. The observed variations appear quite similar at 15 and $230 \mathrm{GHz}$ bands, the most densely sampled frequencies. Faster variations (for instance, the flare between 3 and 4) superimposed on the broad outburst can be seen here. A prominent flare is also apparent in the $86 \mathrm{GHz}$ light curve; however, the data sampling is quite sparse close to the peak of the outburst. The data sampling is even worse at $350 \mathrm{GHz}$, and as a result, the flaring activity is less evident.

The PP (top) and EVPA (bottom) curves at the 86 and $230 \mathrm{GHz}$ radio bands are shown in Figure 9. There are significant variations in the $230 \mathrm{GHz}$ band PP curve. For instance, the PP drops from $15 \%$ to $5 \%$ between $\mathrm{JD}^{\prime}=1600$ and 1700, and it increases again to $15 \%$ at $\mathrm{JD}^{\prime} \sim 1720$. The observed polarization was quite high $(\sim 15 \%)$ for the rest of the period. On the other hand, the PP variations at the $86 \mathrm{GHz}$ band and the EVPA variations at both the 86 and the $230 \mathrm{GHz}$ bands were quite modest $\left(\Delta \chi \leqslant 25^{\circ}\right)$.

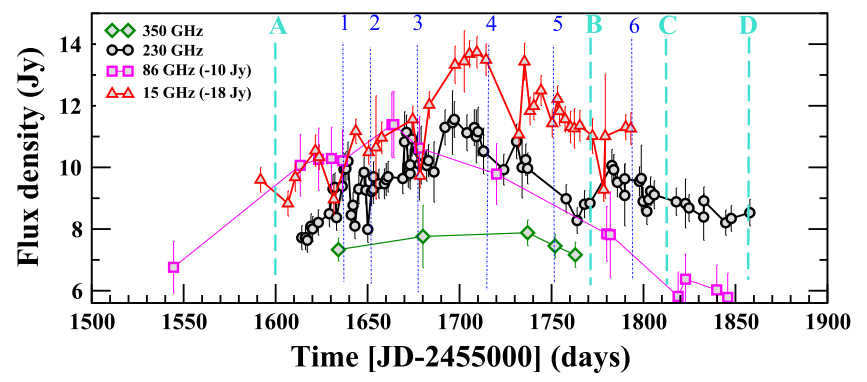

Figure 8. Flux density light curves at the $15,86,230$, and $350 \mathrm{GHz}$ radio bands (vertical dotted-dashed and dashed lines are the same as in Figure 1). The 15 and $86 \mathrm{GHz}$ data shifted by -18 and $-10 \mathrm{Jy}$, respectively.

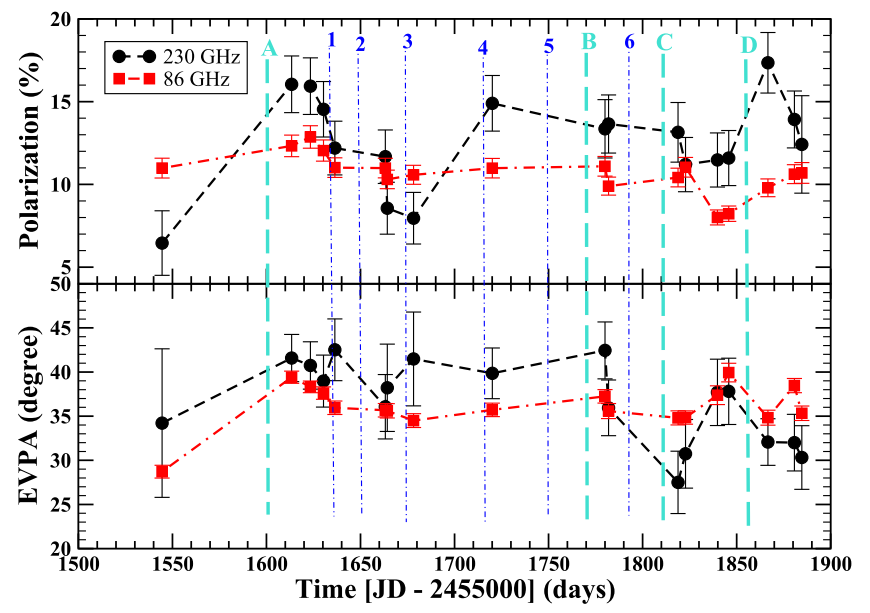

Figure 9. PP (top) and EVPA (bottom) curves at the 86 (red) and 230 (black) $\mathrm{GHz}$ bands (vertical dotted-dashed and dashed lines are the same as in Figure 1).

\subsection{Light-curve Analysis}

\subsubsection{Fractional Variability}

To quantify how the amplitude of flux variations changes as a function of frequency, we compared the fractional variability amplitude $\left(F_{\text {var }}\right)$ of the observed light curves. For a given light curve with an average flux $\bar{x}$ and sample variance $S^{2}, F_{\text {var }}$ is defined as (Vaughan et al. 2003)

$$
F_{\mathrm{var}}=\sqrt{\frac{S^{2}-\overline{\sigma_{\mathrm{err}}^{2}}}{\bar{x}^{2}}},
$$

where $\overline{\sigma_{\text {err }}^{2}}$ is the mean of the squared measurement uncertainties. The uncertainty on $F_{\text {var }}$ due to the measurement-error fluctuations was derived through Monte Carlo simulations by Vaughan et al. (2003, Equation (B2)).

Figure 10 shows the calculated $F_{\text {var }}$ at different frequencies. A gradual increase in $F_{\text {var }}$ with frequency is visible in the radio regime, and this trend continues until the IOU regime. The first

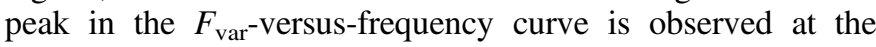
optical $U$ passband, which is followed by a dip in the X-ray regime. Compared to the lower frequencies, the $F_{\mathrm{var}}$ is extremely large at $\gamma$-rays; $F_{\text {var }}>1$ implies more than $100 \%$ variations.

\subsection{Parsec-scale Jet Kinematics}

In Figure 11, we display an example image of the 3C 279 jet. The top panel shows the circular Gaussian components 


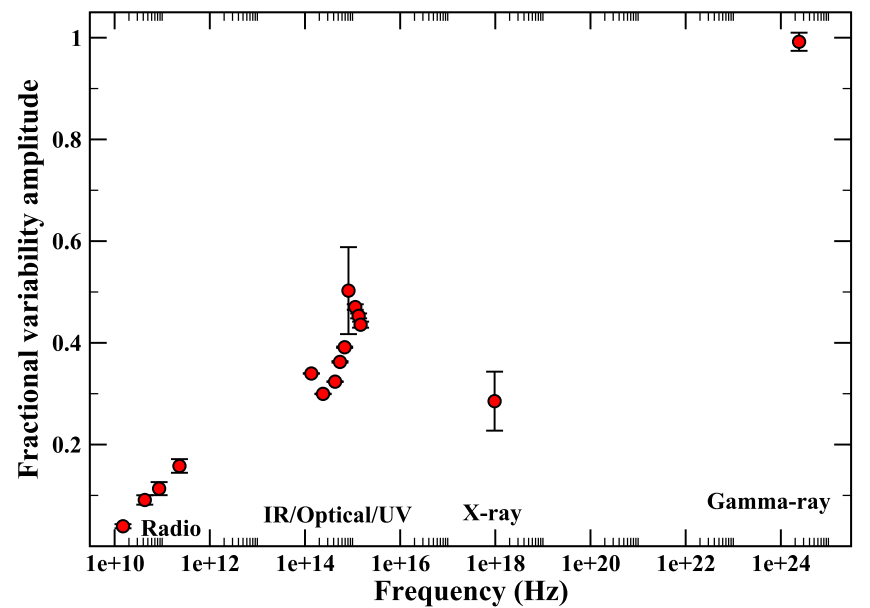

Figure 10. Fractional variability amplitudes of $3 \mathrm{C} 279$ from radio to $\gamma$-ray energies.

(yellow circled crosses) superimposed on the total intensity contours. The bottom panel shows the composite superresolved image convolved with a beam size of 0.1 mas $(0.63 \mathrm{pc})$. In the composite image, the contours represent the total intensity, and the color scale represents the polarized intensity. The yellow line segments mark the EVPA direction.

The total intensity of the jet is described using circular Gaussian components, and we list the model fit parameters in Table 2 in the appendices. To study the evolution of flux density and the polarization of components, as well as the component motion and its evolution as a function of time, we cross-identify the features using the following assumption. We assume that the changes in the flux density, distance from the VLBI core, position angle, and size should be small for the time period between adjacent epochs to prevent a potentially large systematic error arising from the incorrect crossidentification.

\subsubsection{Component Motion}

The VLBI core centered at R.A. 1256 11.167, decl. -05 47 21.53 is chosen as a reference point and is fixed to coordinates $(0,0)$ to study the kinematics of individual components. The absolute position of the core could change because of changes in opacity, pressure, or instabilities in the jet (see Hodgson et al. 2017 and Lisakov et al. 2017 for details). However, whether we fix the core position to $(0,0)$ or leave it free does not affect the relative separation of the components with regard to the core. During the course of our campaign period, we identified a total of six components- $\mathrm{C} 1, \mathrm{C} 2, \mathrm{C} 3, \mathrm{NC} 1, \mathrm{NC} 2$, and $\mathrm{NC} 3$ - in addition to the core. Figure 12 shows the evolution of the radial distance of the components from the core (a) and their trajectories in the $X-Y$ plane ${ }^{25}$ projected on the sky (b). The flux density variations in the individual knots are shown in Figure 12(c).

In 2013 August, the jet's brightness could be well described using five components ( $\mathrm{C} 1-\mathrm{C} 3$ and two unidentified components) in addition to the core (see Table 2 in the Appendices). A new component (NC1) appeared in 2013 November, followed by the ejection of two more components in 2014 May (NC2)

\footnotetext{
${ }^{25}$ Here $X=r \cos \left(\theta+90^{\circ}\right)$ and $Y=r \sin \left(\theta+90^{\circ}\right) ; r$ is the radial separation from the core and $\theta$ is the position angle with respect to an imaginary northsouth line drawn through the map center.
}

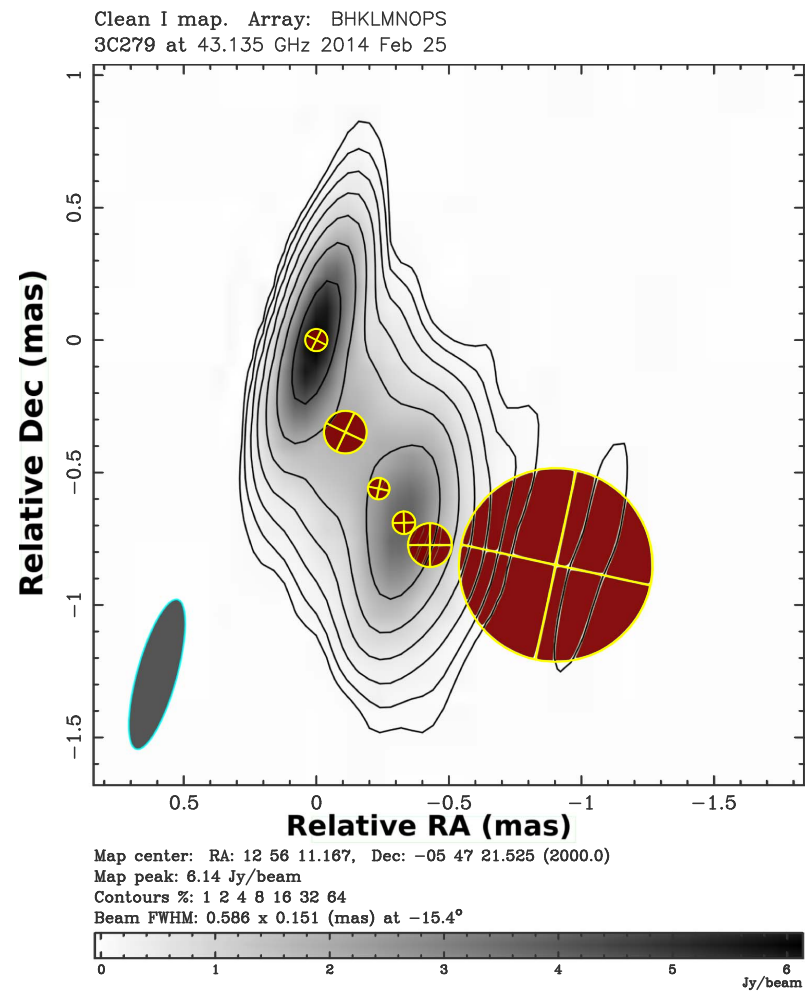

PLot file version 2 created 09-JUN-2016 16:29:18 GREY: 3C279 PPOL 43135.375 MHz FEB14 PI.FITS.1 ICONT:3C279 IPOL 43135.375 MHZ FEB14 I.FITS.1

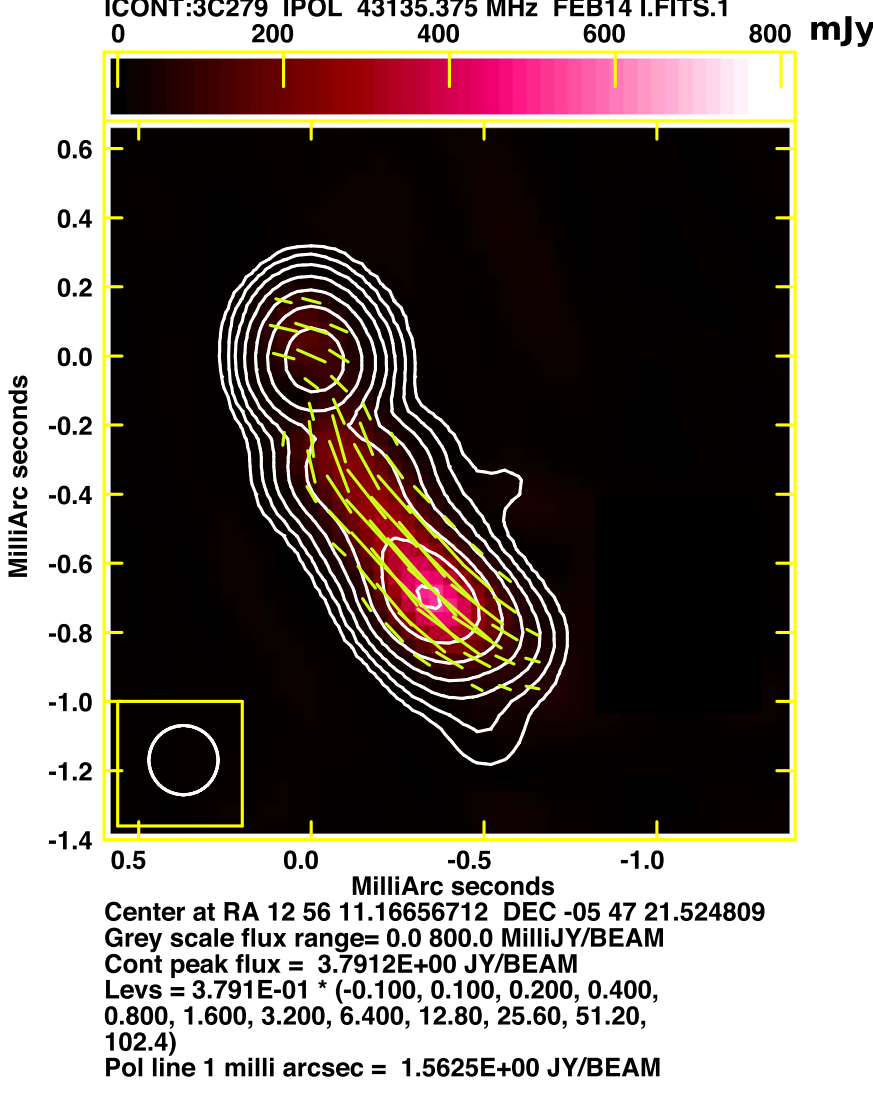

Figure 11. Top: example image of 3C 279 as observed in 2014 February, with Gaussian circular components plotted on top of the total intensity contours. Bottom: composite total (contours) and polarized (color) intensity image of 3C 279 convolved with a beam size of 0.1 mas. The line segments (the length of the segments is proportional to the fractional polarization) show the EVPA direction. The ellipse/circle in the bottom left corner is the synthesized beam of the array. 

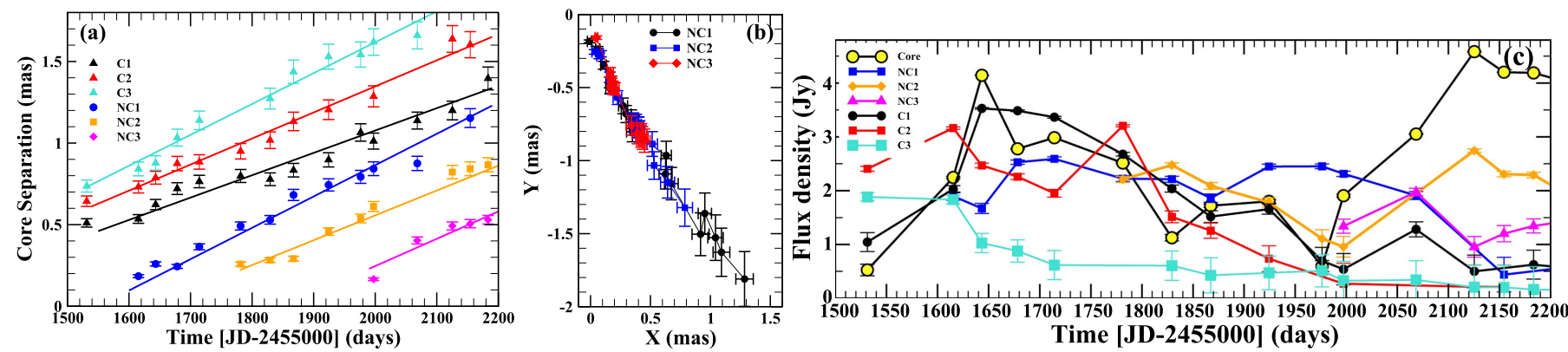

Figure 12. (a) Temporal evolution of the components' radial separation from the core; the solid lines represent fits with a linear function. (b) Trajectories of the components in the $X-Y$ plane (see Section 3.3.1 for details). (c) Flux density variations of the individual knots.

Table 1

Kinematical Parameters of the Jet Emission

\begin{tabular}{lccc}
\hline \hline Component & $\mu\left(\mathrm{mas} \mathrm{yr}^{-1}\right)$ & $\beta_{\text {app }}(\mathrm{c})$ & $T_{0}$ (days) \\
\hline $\mathrm{C} 1$ & $0.49 \pm 0.04$ & $15.8 \pm 1.2$ & $\ldots$ \\
$\mathrm{C} 2$ & $0.58 \pm 0.03$ & $18.4 \pm 0.8$ & $\ldots$ \\
$\mathrm{C} 3$ & $0.69 \pm 0.02$ & $21.8 \pm 0.7$ & $\ldots$ \\
$\mathrm{NC} 1$ & $0.71 \pm 0.02$ & $22.4 \pm 0.7$ & $1558_{+15}^{-28}$ \\
$\mathrm{NC} 2$ & $0.55 \pm 0.03$ & $17.5 \pm 0.8$ & $1638_{+40}^{-27}$ \\
$\mathrm{NC} 3$ & $0.61 \pm 0.04$ & $19.2 \pm 1.2$ & $1810_{+26}^{-19}$ \\
\hline
\end{tabular}

Note. $\mu$ : angular speed. $\beta_{\text {app }}$ : apparent velocity. $T_{0}$ : component's core separation time.

and December (NC3). The core brightened before the ejection of a new component, and later the component appeared in the jet. All of these components exhibit significant motion, especially in the radial direction. As seen in Figure 12(b), the components tend to follow the same linear trajectories in the $X-Y$ plane up to a distance of $\sim 0.5$ mas. This is in contrast to what we usually observe for blazars. Wiggly/curved trajectories of components are often seen for blazars (see Chatterjee et al. 2008; Lister et al. 2013, Rani et al. 2014; 2015; and references therein).

To study the component motion in the radial direction, we fitted the radial separation of the components relative to the core using $r(t)=\mu \times\left(t-T_{0}\right)$, where $\mu$ is the angular speed in mas $\mathrm{yr}^{-1}$ and $T_{0}$ corresponds to the ejection time of the components from the core. The solid lines in Figure 12(a) represent the best-fit functions. The fit provides the angular speed $(\mu)$, which we used to calculate the apparent velocity following $\beta_{\text {app }}=\frac{\mu \cdot D_{L}}{c .(1+z)}$, where $D_{L}$ is the luminosity distance and $z$ is the redshift of the source. In Table 1 , we list the angular and apparent speeds of the components. Back-extrapolation of the components' motion is used to estimate the ejection time of the new components (NC1-NC3). The calculated ejection times of the new components are listed in Table 1. The highest detected apparent speed $\left(\beta_{\text {app }}\right)$ can be used to get an estimate of the lowest possible Lorentz factor, $\Gamma \geqslant\left(1+\beta_{\text {app }}^{2}\right)^{0.5}$, and maximum viewing angle, $\theta_{\text {view }} \leqslant \sin ^{-1}\left(1 / \beta_{\text {app }}\right)$. Using $\beta_{\text {app }}=$ 22.4 , we get $\Gamma \geqslant 22.4$ and $\theta_{\text {view }} \leqslant 2.6$.

\subsection{Polarization Variations}

Figure 13 presents a comparison of the variations observed in PP at optical and radio frequencies (the left $y$-scale is for the optical data, while the right is for the radio data). Although it is difficult to make a one-to-one comparison between the PP variations at the two frequencies because of sparse sampling of
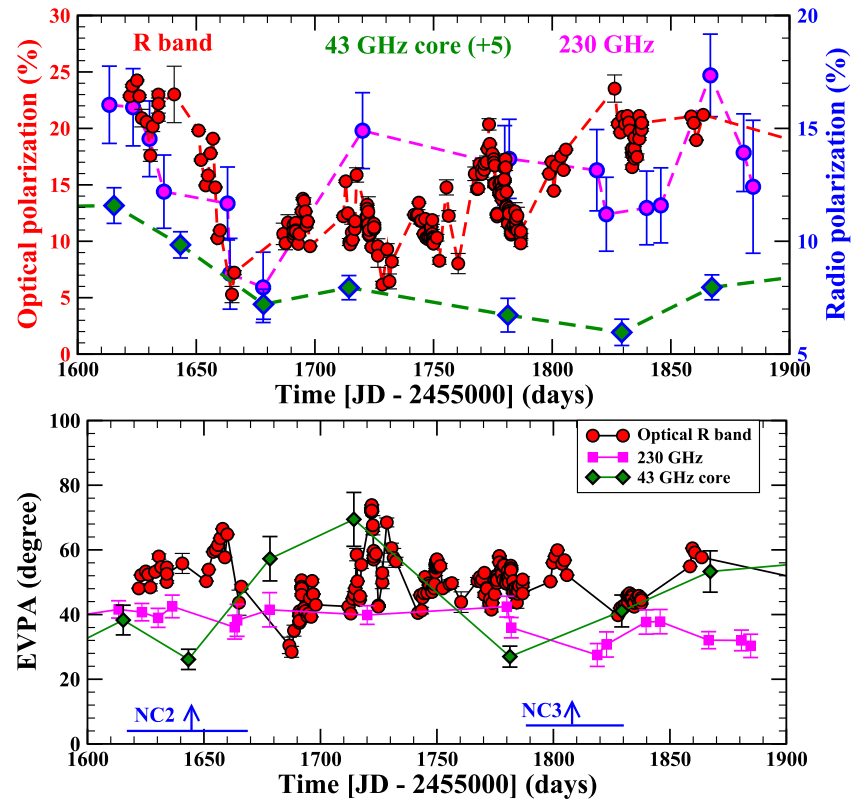

Figure 13. Top: comparison of the PP variations at the radio and optical frequencies. For illustration purposes, the $43 \mathrm{GHz}$ radio core PP curve is shifted by $+5 \mathrm{Jy}$ (the left scale is for the optical data, and the right is for the radio data). EVPA variations are displayed in the bottom panel.

the radio data, we do see a similarity. For instance, in the beginning, the PP is high at both the optical and radio bands. A drop in PP (between $\mathrm{JD}^{\prime} 1600$ and 1680) is observed almost simultaneously at the two frequencies. An increase in PP appears after the dip observed in the optical and $230 \mathrm{GHz}$ data but not in the $43 \mathrm{GHz}$ VLBI data. The PP in the core region remains rather constant after the dip. On the other hand, the PPs at the optical and $230 \mathrm{GHz}$ radio frequencies continue rising until they regain their initial value at the end of the observations.

Figure 13 (bottom) shows a comparison of the EVPA variations at the optical and radio frequencies. In contrast to PP, EVPA variations are rather modest. A peak-to-peak (minimumto-maximum) change of $\sim 60^{\circ}$ is observed in the optical and $43 \mathrm{GHz}$ VLBI data, and in the $230 \mathrm{GHz}$ data, it is $\leqslant 25^{\circ}$. The average EVPA values at the three frequencies are also quite similar $\left(\sim 40^{\circ}-60^{\circ}\right)$. This indicates that the optical and radio polarization angles (EVPAs) are roughly parallel to the jet direction $^{26}\left(\sim-135^{\circ}\right)$. We noticed a change in the VLBI core

\footnotetext{
${ }^{26}$ Since at optical frequencies, we are already in the optically thin regime, the Faraday effects are negligible here.
} 


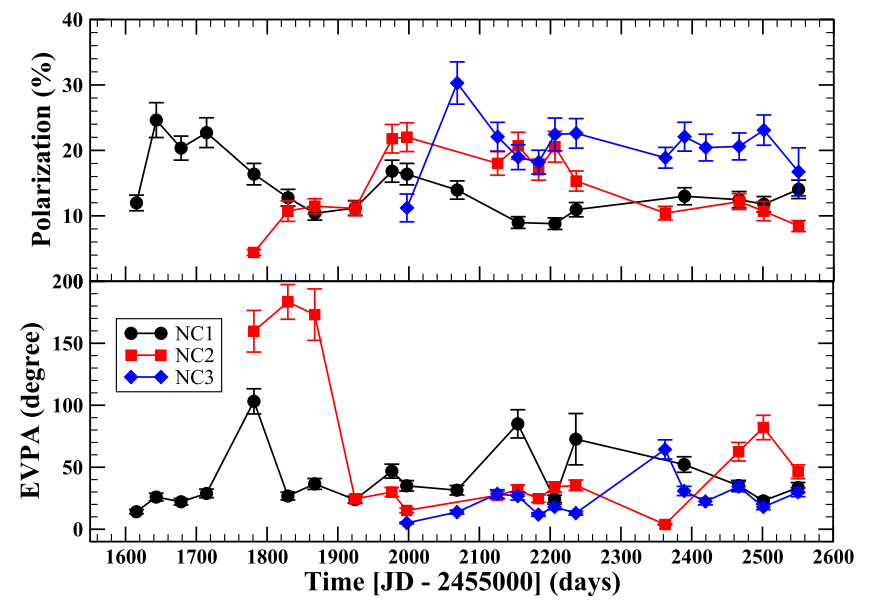

Figure 14. PP and EVPA variations observed in the individual components.

EVPA during the ejection of the new components (NC2 and NC3), which could be because of a different EVPA orientation of the new component(s).

The PP (top) and EVPA (bottom) variations observed in the new ejected components are shown in Figure 14. The PPs of the components gradually increase after their separation from the core and stay as high as $\geqslant 20 \%$. This could result from either a transition from the optically thick to thin regime at $43 \mathrm{GHz}$ or a progressive ordering of the magnetic field with distance down the jet. The EVPAs of the new components (especially NC2) show some modest variations but still remain aligned along the jet axis.

\subsection{Flux Density versus Polarization Variations}

In Figure 15, we show a comparison of optical flux density and polarization variations. We used an inverse $y$-scale for the optical PP data. During the first three $\gamma$-ray flares (1-3), the optical flux is rising, and a decay is seen in the PP. The peaks of flares 4 and 5 coincide with the dips in the PP curve, indicating a clear anticorrelation between the two. The anticorrelation is even more evident in the PP-versus-optical flux plot on the right. A formal linear Pearson correlation test suggests a significant anticorrelation between the optical flux and polarization data; we obtained $r_{\mathrm{P}}=-0.76$ at the $>99.99999 \%$ confidence level.

A comparison of the optical PP and $\gamma$-ray photon flux variations is shown in Figure 16 (left; again, an inverse scale is used for the optical PP data). There are hints of an apparent anticorrelation between the two. For instance, at the onset of the $\gamma$-ray outburst $\left(\mathrm{JD}^{\prime} \sim 1610\right)$, the optical PP is quite high $(\sim 25 \%)$. Also, the peaks of the $\gamma$-ray flares (4 and 5) roughly coincide with the dips in the PP curve. Finally, the optical PP regained its original value $(\sim 25 \%)$ at the end of the outburst $\left(\mathrm{JD}^{\prime} \sim 1820\right)$. The anticorrelation is even more evident in the optical PP-versus- $\gamma$-ray photon flux plot shown in the right panel of Figure 16. The statistical significance is tested via the linear Pearson correlation analysis, which suggests a significant $\left(r_{\mathrm{P}}=-0.27\right.$ at a confidence level $\left.=98.5 \%\right)$ anticorrelation between the two.

Figure 17 shows the PP-versus-spectral index plot. We again used the linear Pearson correlation analysis to test the significance of the correlation between PP and optical $/ \gamma$-ray spectral indices. Formally, we obtained $r_{\mathrm{P}}=0.63$ at the
99.9998\% confidence level for the PP-versus- $\gamma$-ray photon index data (see top panel), which suggests a significant correlation between the two. The PP-versus-optical spectral index plot suggests a correlation $\left(r_{\mathrm{P}}=0.57\right)$ at the $93.7 \%$ confidence level. A positive correlation between PP and optical $/ \gamma$-ray spectral indices indicates that higher PP is observed for a steeper spectrum. A significant hardening of the optical $/ \gamma$-ray spectrum is observed during bright flares (see Sections 3.1.1 and 3.1.3 for details).

A change in PP could be simply explained by a change in either the B-field order (i.e., an increase in PP indicates a more ordered magnetic field) or the spectral index of the emitting particles. We could expect to see some increase in PP due to steepening of the electron spectrum or synchrotron spectrum (degree of maximum polarization $=(\alpha+1) /(\alpha+5 / 3)$, where $\alpha$ is the spectral index). For instance, steepening of a spectrum by one magnitude $(\Delta \alpha=1)$ produces a $\leqslant 5 \%$ increment in PP magnitude. Although the PP significantly correlates with $\alpha$, our observations suggest variations of $20 \%(\Delta P P=20)$. This, in turn, suggests that the PP variations cannot be solely explained via steepening of the spectrum, as a change in the spectral index has only a very slight effect on the PP.

Since the mean polarization is the maximum divided by the square root of the number of cells ${ }^{27}$ involved in the emission, the observed anticorrelation between $\gamma$-ray flux and optical PP could be because of the presence of more turbulent cells during the outburst than at other times. The jet plasma is highly dynamic; plasma instabilities (e.g., the current-driven kink instability and/or magnetohydrodynamical instabilities; Nalewajko \& Begelman 2012; Nishikawa et al. 2016) could occur and vary with time, which basically drives the turbulence. In short, instabilities drive turbulence, which can influence the number of cells in a given emission region. This, in turn, implies that the turbulence could enhance the efficiency of particle acceleration, which is why there is an outburst and also why the spectrum is flatter during the outburst and steeper before and after the outburst when the PP is higher. An observational test of the turbulence cell model (TEMZ; Marscher 2014) is to compare the standard deviation of random PP fluctuations (compared to the systematic variations). In case of the presence of only turbulent component(s), $\sigma(\mathrm{PP})=0.5\langle\mathrm{PP}\rangle$. We used an equal time bin when the PP is not changing systematically to determine $\sigma(\mathrm{PP})$. For $3 \mathrm{C} 279$, the calculated $\sigma(\mathrm{PP})=(0.08-0.25)\langle\mathrm{PP}\rangle$, which suggests the presence of a polarized steady component as well or the presence of multiple emission components with different polarizations.

To further test the hypothesis of the presence of a steady polarized component in addition to the multiple turbulent components, we investigate the variations in Stokes $Q$ and $U$ parameters. Photometric and polarimetric measurements obtained on the same day were used to calculate the fractional Stokes parameters, $Q_{\text {frac }}=$ PP $\cos (2 \times$ EVPA $)$ and $U_{\text {frac }}=$ PP $\sin (2 \times$ EVPA). Figure 18 (left) shows the variations in optical $R$-band $Q_{\text {frac }}$ and $U_{\text {frac. }}$ A clear difference in the Stokes $Q_{\text {frac }}$ and $U_{\text {frac }}$ variations can be noticed here. The observed dependence between the Stokes parameters is given in the right panel of Figure 18 (red: optical $R$ band; magenta: $43 \mathrm{GHz}$ radio core; blue: $230 \mathrm{GHz}$; green: $86 \mathrm{GHz}$ ). The $Q_{\text {frac }}, U_{\text {frac }}$ centroid clearly deviates from $(0,0)$. We noticed a similar shift at the 86

\footnotetext{
27 The smallest-scale emission region having a preferred magnetic field orientation (Marscher 2014).
} 

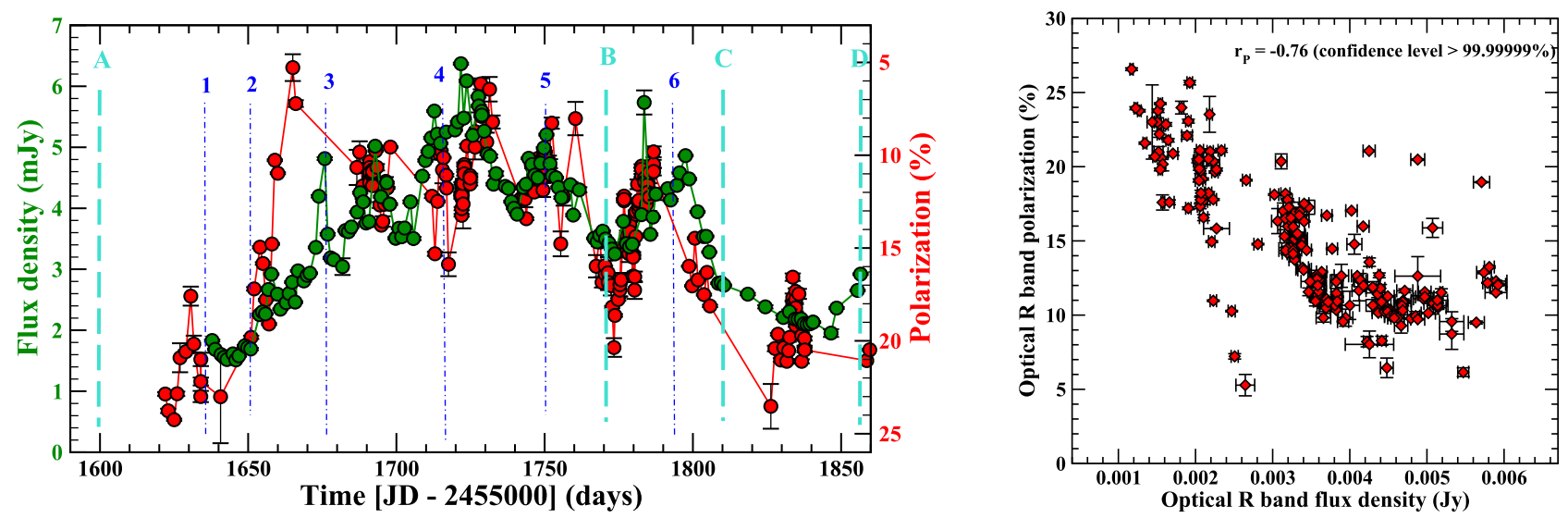

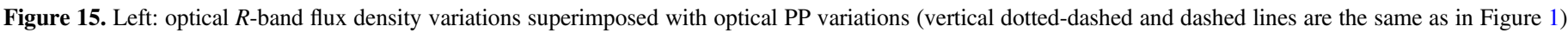
The right $y$-scale is for optical polarization and is reversed for comparison purposes. Right: optical polarization vs. flux density plot.
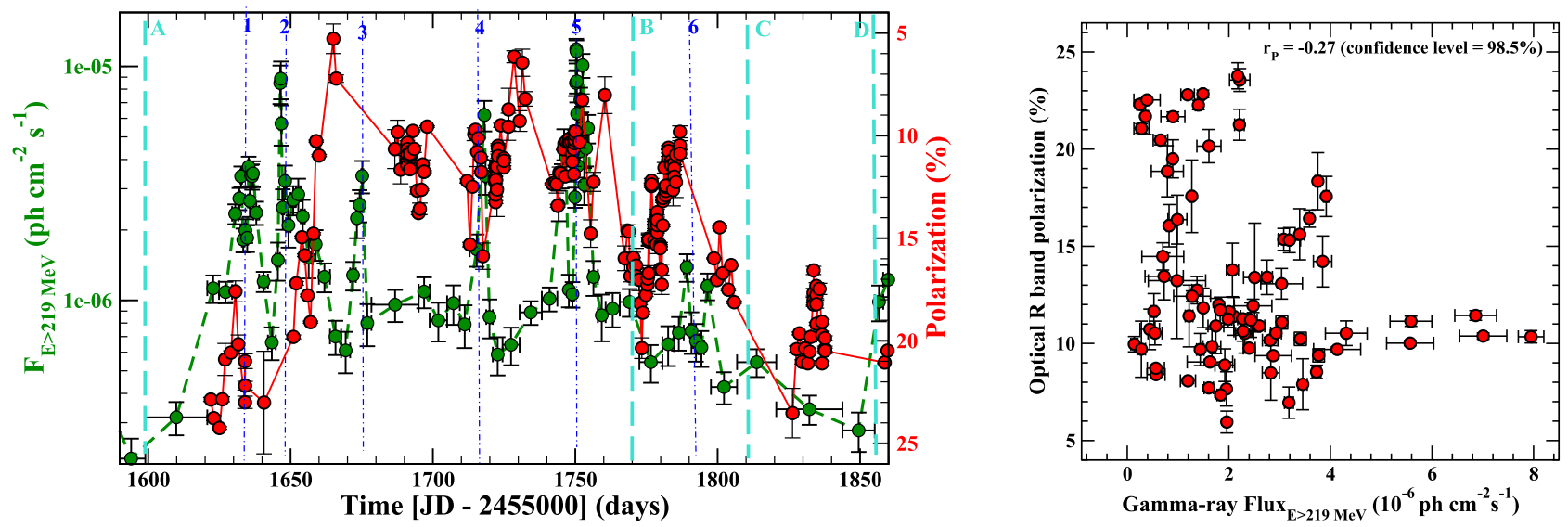

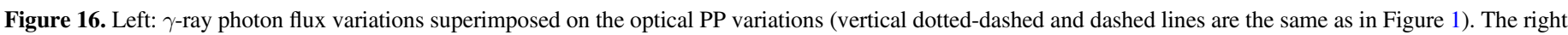
$y$-scale is for optical polarization and is reversed for comparison purposes. Right: optical polarization vs. $\gamma$-ray photon flux plot.

and $230 \mathrm{GHz}$ radio bands and in the $43 \mathrm{GHz}$ radio core. The observed shift of the $Q, U$ centroid with respect to $(0,0)$ can be explained by the presence of a steady component with a polarization direction aligned along the jet axis (Blinov \& Hagen-Thorn 2009). Moreover, a similarity of EVPA values at optical and radio frequencies with the VLBI core EVPAs supports the alignment of optical/radio polarization direction along the jet axis (Section 3.4). As suggested by Blinov \& Hagen-Thorn (2009), if the steady polarized component has a higher degree of polarization, one can expect to see an anticorrelation between the flux and degree of polarization. The observed PP at the beginning/end of the outburst (see Figures 15 and 16 ) is $\sim 25 \%$, which means that the steady polarized component is higher than $25 \%$. A steady polarized component having its direction parallel to the jet axis could be the toroidal magnetic field component of the jet. Alternatively, the presence of multiple emission components with different polarizations could be plausible as well.

\subsection{Connection between Jet Activity and Broadband Emission}

Given the superposition of multiple modes of flaring activity, it is quite challenging to understand the broadband flaring behavior of the source. The major outburst (A-D) is accompanied by faster flares across the whole electromagnetic spectrum (see Figure 1). Interestingly, a new component, "NC2," is ejected from the core during the period of the first three (1-3) $\gamma$-ray flares. The next three flares (4-6) were followed by a continuous decay in the source brightness at the $\gamma$-ray, optical, and radio frequencies between periods $\mathrm{C}$ and $\mathrm{D}$. Later, the ejection of a new component "NC3" from the core suggests a delay between the high-energy activity and the component's core-separation timing.

Simulations (as described in the appendices) were used to test whether or not the connection between component ejection and $\gamma$-ray flaring activity is just by chance. First, we simulated a total of 1000 light curves. Next, we checked how often we see a $\gamma$-ray flare in the simulated data during the ejection of $\mathrm{NC} 2\left(\mathrm{JD}^{\prime}=1611-1678\right)$ and $\mathrm{NC} 3\left(\mathrm{JD}^{\prime}=1790-1836\right)$. Given the superposition of multiple modes of flaring activity, defining a flare could be tricky. We choose a simplistic approach here. As the typical duration of the rapid $\gamma$-ray flares (1-6) is 10-30 days, we define a continuous rise in the $\gamma$-ray flux above $6.6 \times 10^{-7}$ photons $\mathrm{cm}^{-2} \mathrm{~s}^{-1}$ (two times above the quiescent level) for $\geqslant 20$ days as a flare and tested how often this condition is met in our simulated light curves. During the NC2 

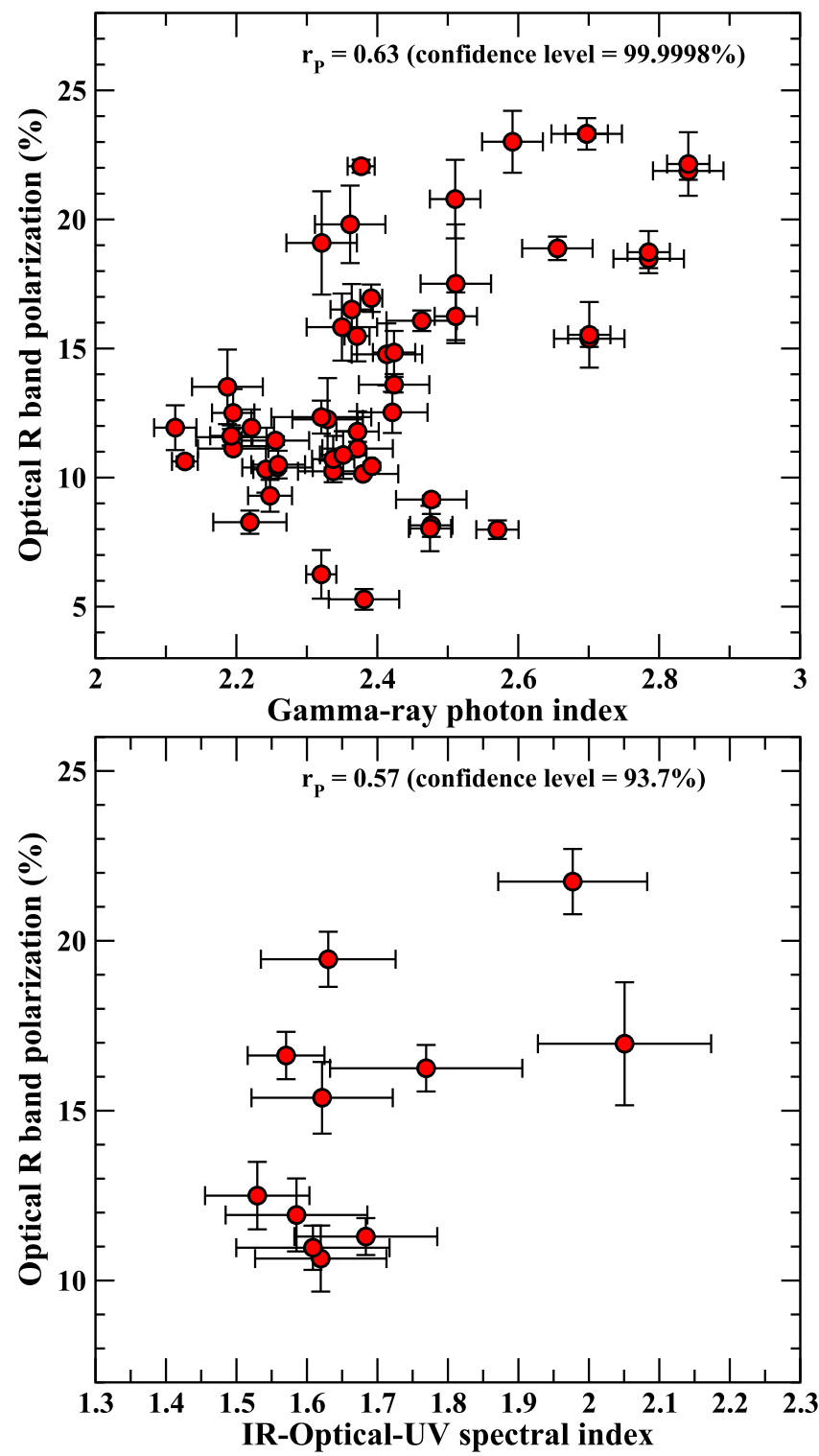

Figure 17. Optical polarization vs. $\gamma$-ray photon index (top) and IOU spectral index (bottom).

ejection period, we found a flare in 28 out of 1000 cases; in short, the ejection of $\mathrm{NC} 2$ is connected to the $\gamma$-ray flaring activity with a $97.2 \%$ confidence level. Similarly, we found that the $\gamma$-ray flares are related to NC3 with a $95.8 \%$ confidence level. We also tested the significance of connection between $\gamma$-ray flares observed within 50 days before and after the ejection of the components. Our calculations suggest that the chance coincidence of $\mathrm{NC} 2$ being associated with a flare(s) 50 days before or after its ejection is below $90 \%$. In the case of $\mathrm{NC} 3$, there are fair chances $(94.2 \%)$ that its ejection could be related to a flare(s) observed 50 days prior to the ejection, while the confidence level of its ejection being connected to a flare observed with 50 days after its ejection is below $90 \%$. Moreover, we tested the chance coincidence for the different flare durations. For a short flare duration of 10 days, the probability of observing a flare by chance during the ejection of
$\mathrm{NC} 2$ (and NC3) is less than 10\%; i.e., the confidence level of $\mathrm{NC} 2$ (and NC3) being associated with a $\gamma$-ray flare is $\simeq 90 \%$. In the case of a long-duration flare (30 days), the confidence level is $>96 \%$.

\subsection{Multiple Energy Dissipation Regions}

During 2013-2014, we observed two modes of flaring activity (faster flares sitting on top of the long-term outburst) in 3C 279 at radio, optical and $\gamma$-ray energies. Although X-ray counterparts were observed for two flares, the long-term outburst seems to be missing. The broadband spectral energy distribution modeling during this period (reported in Hayashida et al. 2015) suggests that single-zone emission models that matched the optical and $\gamma$-ray spectra failed to describe the $\mathrm{X}$-ray emission, which suggests the presence of multiple emission regions.

Unlike the last three flares (4-6), the first three $\gamma$-ray flares (1-3) are missing their optical/radio counterparts, except for flare 3, which is observed at optical bands. Moreover, the IOU spectrum is relatively softer $\left(\alpha_{\text {IOU }} \sim\right.$ 1.8-2.0) during the first episode of flares. Later, the IOU spectrum gets harder $\left(\alpha_{\mathrm{IOU}} \sim 1.6\right)$. A new component, NC2, appeared in the jet during flares 1-3. Ejection of NC2 during the first three flares indicates that these high-energy flares have to be produced either at the VLBI core or very close to the core region; otherwise, we would have observed a delay between the high-energy flares and the component ejection, which we did not. A delay is observed between the last three flares (4-6) and the ejection of NC3. This indicates that the high-energy region is located further upstream of the VLBI core (closer to the central black hole). Moreover, the PP-versus- $\gamma$-ray/optical flux and spectral index correlation (discussed in Section 3.5) suggests the presence of multiple turbulent cells in a given emission region and that the number of these cells is variable with time.

We interpret the observed broadband flaring activity in the following manner (see Figure 19). A disturbance at the base of the jet could cause a shock wave and propagate down the jet and could be related to the onset of the major outburst (Figure 19(a)). Later, the moving shock reaches the $43 \mathrm{GHz}$ core, producing the first three flares (1-3). Given the different B-field properties of cells, the particle acceleration efficiency could be different for different cells. Since what we observe at a given frequency is their collective emission, concurrent broadband flaring activity may or may not be observed. The moving shock disturbs the jet outflow and becomes knot $\mathrm{NC} 2$ as it separates from the core (Figure 19(b)). In the second episode of flaring activity (4-5), the emission region seems to be located further upstream of the core (closer to the central black hole; see Figure 19(c)). Later, the disturbance/shock wave passes the core region, resulting in the ejection of NC3 (Figure 19(d)). Our analysis therefore supports two possible energy dissipation sites in 3C 279. The location of the dissipation zone depends on the parameters of a disturbance, especially the Lorentz factor $(\Gamma)$; for disturbances with lower $\Gamma$, the dissipation zone is expected to be closer to the black hole than for those with larger $\Gamma$ (Katarzyński \& Ghisellini 2007). Although the values of $\beta_{\mathrm{app}}$ of $\mathrm{NC} 2$ and NC3 are similar within the uncertainties, it is possible that $\Gamma_{\mathrm{NC} 2}>\Gamma_{\mathrm{NC} 3}$ that 

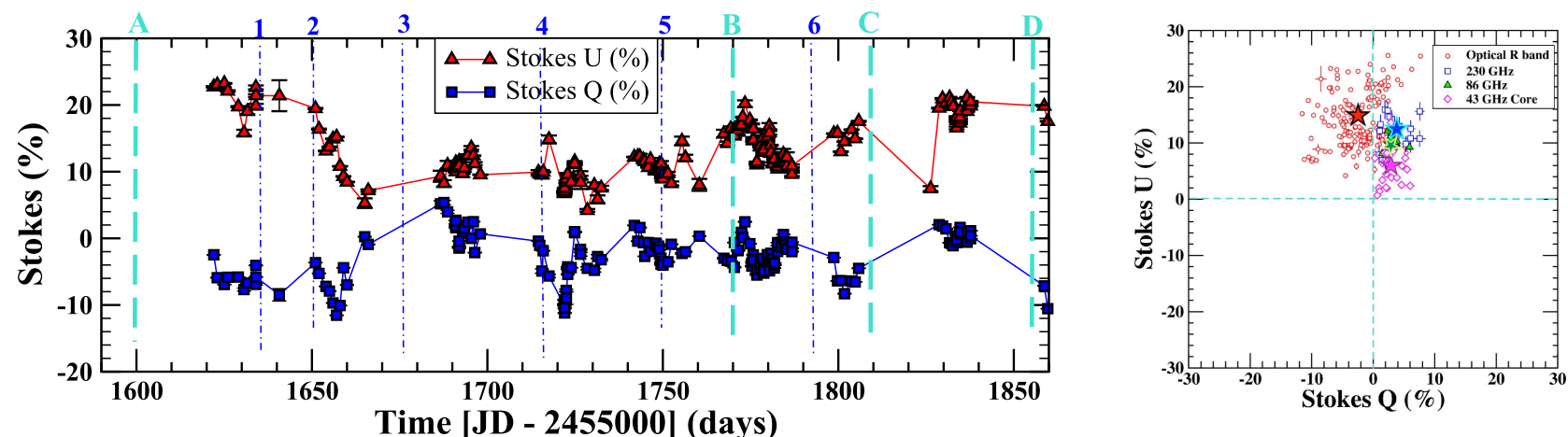

Figure 18. Left: optical $R$-band $Q_{\text {frac }}$ and $U_{\text {frac }}$ variations (vertical dotted-dashed and dashed lines are the same as in Figure 1). Right: Stokes $Q_{\text {frac }}$ vs. $U_{\text {frac }}$; the centroid in each case is marked with a star.

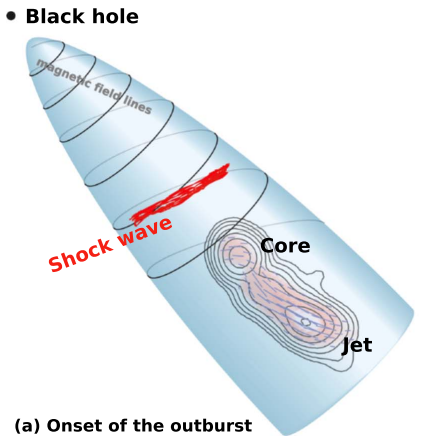

(a) Onset of the outburst

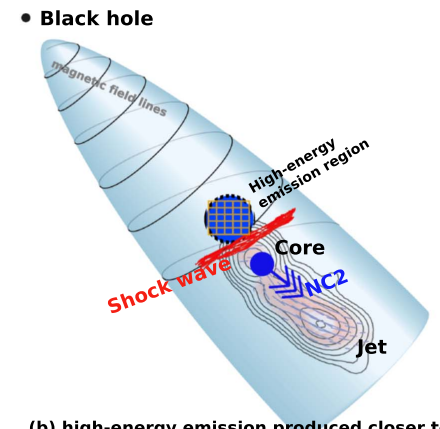

(b) high-energy emission produced closer to the core and followed by the ejection of NC2

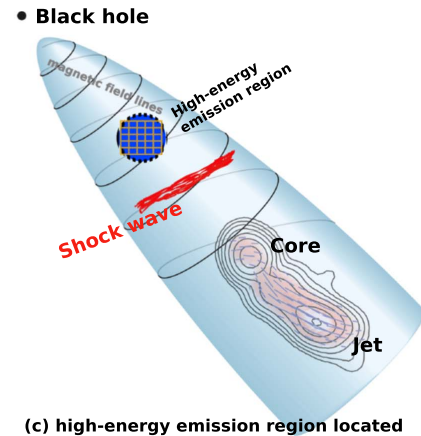

closer to the black hole

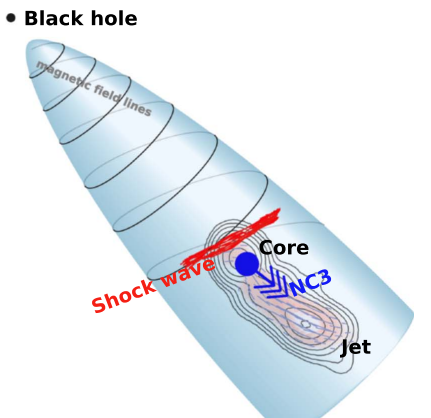

(d) disturbance reaches the core and NC3 is ejected

Figure 19. Sketch of the proposed scenario in 3C 279 (not to scale). A disturbance (or shock wave) reaches the core (a), producing the high-energy flares (1-3) closer to the core region and an ejection of component NC2 (b). (c) The high-energy emission region is located closer to the black hole, and later, the disturbance passes the core, resulting in the ejection of NC3 (d).

would result in the dissipation zone during flares $1-3$ being farther from the black hole and closer to the VLBI core. Both observations (Jorstad et al. 2005; Rani et al. 2015; Hodgson et al. 2017) and simulations (Gómez et al. 1997; Fromm et al. 2012) support the presence of standing shocks in parsec-scale AGN jets. In fact, at short mm-radio bands, the VLBI core could be a standing shock as well (Marscher 2008; Hodgson et al. 2017). Passage of a moving shock through standing shocks is quite efficient in accelerating particles to high energies, which could explain why some sites are more favored than others for energy dissipation (Gómez et al. 1997; Meyer et al. 2015).

\section{Summary and Conclusion}

We used high-frequency VLBI observations to probe the broadband flaring activity in $3 \mathrm{C} 279$. At $\mathrm{GeV}$ energies, the source went through a series of flares superimposed on a longterm outburst from $\mathrm{JD}^{\prime}=1600$ to 1850 (2013 November to 2014 August). In total, we observed six $\gamma$-ray flares, and four of these flares have optical (and radio) counterparts. X-ray counterparts are only observed for two flares (2 and 5). Moreover, similar to $\gamma$-rays, we observed two different modes of flaring activity (fast flares superimposed on a long-term outburst) at optical and radio frequencies. On the other hand, the long-term outburst activity seems to be missing at X-rays.
Two modes (fast flares superimposed on long-term variations) of variability are also observed in PP data at optical and radio frequencies. On the other hand, the polarization angle (EVPA) variations are quite modest at both the optical and radio frequencies, and the EVPAs are well aligned with the jet direction.

Two new components (NC2 and NC3) were ejected from the core during the flaring activity period and are connected to the source flaring activity with $\geqslant 95 \%$ probability. NC2 appeared in the jet during the first three flares (1-3) pointing to the $43 \mathrm{GHz}$ VLBI core as the possible location of energy dissipation. Ejection of NC3 happened after the last three flares (4-6), which puts the location of energy dissipation further upstream of the VLBI core (in a region closer to the black hole). Our analysis therefore argues in favor of multiple energy dissipation regions in the source.

We detect an anticorrelation between the optical PP and $\gamma$-ray/optical flux variations, while a positive correlation is seen for the PP and $\gamma$-ray/optical spectral index variations. We propose that this could be explained using the turbulent multi-zone cell model (TEMZ). If there are more turbulent cells in the emission region during the outburst than other times, one might expect to see an anticorrelation between $\gamma$-ray/optical flux and PP. This, in turn, implies that the turbulence is related to the efficiency of particle acceleration. The observations, however, cannot be solely described using the turbulent component. A shift in the Stokes $(Q, U)$ centroid with regard 
to $(0,0)$ suggests the presence of multiple emission components with different polarizations.

The Fermi/LAT Collaboration acknowledges generous ongoing support from a number of agencies and institutes that have supported both the development and the operation of the LAT, as well as scientific data analysis. These include the National Aeronautics and Space Administration and the Department of Energy in the United States; the Commissariat à l'Energie Atomique and the Centre National de la Recherche Scientifique/Institut National de Physique Nucléaire et de Physique des Particules in France; the Agenzia Spaziale Italiana and the Istituto Nazionale di Fisica Nucleare in Italy; the Ministry of Education, Culture, Sports, Science and Technology (MEXT), High Energy Accelerator Research Organization (KEK), and Japan Aerospace Exploration Agency (JAXA) in Japan; and the K. A. Wallenberg Foundation, Swedish Research Council, and Swedish National Space Board in Sweden. Additional support for science analysis during the operations phase is gratefully acknowledged from the Istituto Nazionale di Astrofisica in Italy and the Centre National d'Études Spatiales in France. This work was performed in part under DOE Contract DE-AC02-76SF00515.

This research was supported by an appointment to the NASA Postdoctoral Program at the Goddard Space Flight Center, administered by the Universities Space Research Association through a contract with NASA. This study makes use of $43 \mathrm{GHz}$ VLBA data from the VLBA-BU Blazar Monitoring Program (VLBA-BU-BLAZAR; http://www.bu. edu/blazars/VLBAproject.html), funded by NASA through the Fermi Guest Investigator Program. The VLBA is an instrument of the Long Baseline Observatory. The Long Baseline Observatory is a facility of the National Science Foundation operated by Associated Universities, Inc. The BU group acknowledges support from US National Science Foundation grant AST-1615796. The Submillimeter Array is a joint project between the Smithsonian Astrophysical Observatory and the Academia Sinica Institute of Astronomy and Astrophysics and is funded by the Smithsonian Institution and the Academia Sinica. This research was supported by an appointment to the NASA Postdoctoral Program at the Goddard Space Flight Center, administered by the Universities Space Research Association through a contract with NASA. KS is supported by the Russian Science Foundation grant 16-12-10481. The St. Petersburg University team acknowledges support from Russian Science Foundation grant 17-12-01029. This research has made use of data from the OVRO $40 \mathrm{~m}$ monitoring program (Richards et al. 2011), which is supported in part by NASA grants NNX08AW31G, NNX11A043G, and NNX14AQ89G and NSF grants AST0808050 and AST-1109911. IA acknowledges support by a Ramón y Cajal grant of the Ministerio de Economía, Industria, y Competitividad (MINECO) of Spain. Acquisition and reduction of the POLAMI data was supported in part by MINECO through grants AYA2010-14844, AYA201340825-P, and AYA2016-80889-P and by the Regional Government of Andalucía through grant P09-FQM-4784. The POLAMI observations were carried out at the IRAM $30 \mathrm{~m}$ telescope. IRAM is supported by INSU/CNRS (France), MPG (Germany), and IGN (Spain). BR acknowledges the help of Roopesh Ojha, Marcello Giroletti, and Dave Thompson for fruitful discussions and comments that improved the manuscript.

\section{Appendix A \\ Testing the Significance of Component Ejections and $\gamma$-ray Flares}

The burst-like $\gamma$-ray flares in 3C 279 do not follow a Gaussian distribution and can be better described via lognormal or $\gamma$ distribution; therefore, we simulated the light curves using the Emmanoulopoulos (Emmanoulopoulos et al. 2013) method implemented by Connolly (2015). The first step is to determine the power spectral density (PSD) slope of the observed $\gamma$-ray light curve. Given that the adaptive binned $\gamma$-ray data is unevenly sampled, we interpolated it using the cubic spline method in $\mathrm{R}^{28}$ As the data is manipulated via interpolation, we tested our PSD result using simulations. We refer to Chidiac et al. (2016) for a step-by-step analysis. We found that the observed source variability can be well described by a PSD slope of $-(0.97 \pm 0.17)$. The method also takes into account the underlying probability density function (PDF) of the given light curve. We simulated a total of 1000 light curves using the best-fit PDF and PSD parameters. The simulated light curves are sampled at the same cadence as the adaptive binned $\gamma$-ray light curve. The average flux and standard deviation of simulated data are equal to that of the observed data. In order to take the adaptive binning to account, the light curves are generated using a variable binning. The "time binning" is a variable parameter in our simulations, and it takes into account the minimum, maximum, and average binning of the observed light curve. Since both the intensity and time binning are independent, free parameters, we have smaller time bins even in low-flux states for several cases. In order to cross-check the significances calculated using this method, we followed an alternative approach. We simulated data with the minimum time bin of the adaptive binned data and sampled the simulated light curves at the same cadence as the adaptive binned $\gamma$-ray light curve. In both cases, we obtained consistent results.

\section{Appendix B Model Fit Component Results}

Table 2 lists the model fit parameters of the 3C 279 jet. The jet kinematic study is presented in Section 3.3. 
Table 2

Results from Gaussian Model Fitting and Component Parameters

\begin{tabular}{|c|c|c|c|c|c|}
\hline $\begin{array}{l}\text { Epoch }\left(\mathrm{JD}^{\prime}\right) \\
\text { Date }\end{array}$ & $\begin{array}{c}S \\
(\mathrm{Jy})\end{array}$ & $\begin{array}{c}r \\
\text { (mas) }\end{array}$ & $\begin{array}{c}\theta \\
(\mathrm{deg})\end{array}$ & $\begin{array}{c}\phi \\
\text { (mas) }\end{array}$ & Comp $^{\mathrm{a}}$ \\
\hline 1531 & $4.52 \pm 0.45$ & $0 \pm 0$ & $0 \pm 0$ & $0.054 \pm 0.005$ & core \\
\hline \multirow[t]{5}{*}{ (2013 Aug) } & $0.52 \pm 0.05$ & $0.28 \pm 0.02$ & $-173 \pm 17$ & $0.092 \pm 0.009$ & $\mathrm{X}$ \\
\hline & $1.04 \pm 0.10$ & $0.51 \pm 0.05$ & $-160 \pm 16$ & $0.074 \pm 0.007$ & $\mathrm{C} 1$ \\
\hline & $2.40 \pm 0.34$ & $0.64 \pm 0.06$ & $-155 \pm 18$ & $0.087 \pm 0.008$ & $\mathrm{C} 2$ \\
\hline & $1.88 \pm 0.18$ & $0.73 \pm 0.07$ & $-153 \pm 15$ & $0.154 \pm 0.015$ & $\mathrm{C} 3$ \\
\hline & $0.38 \pm 0.03$ & $1.13 \pm 0.11$ & $-128 \pm 17$ & $0.693 \pm 0.069$ & $\mathrm{X}$ \\
\hline 1615 & $6.24 \pm 0.62$ & $0 \pm 0$ & $0 \pm 0$ & $0.043 \pm 0.004$ & core \\
\hline \multirow{5}{*}{ (2013 Nov) } & $1.89 \pm 0.18$ & $0.18 \pm 0.01$ & $177 \pm 17$ & $0.109 \pm 0.010$ & $\mathrm{NC} 1$ \\
\hline & $2.02 \pm 0.30$ & $0.53 \pm 0.05$ & $-159 \pm 15$ & $0.136 \pm 0.013$ & $\mathrm{C} 1$ \\
\hline & $3.16 \pm 0.31$ & $0.73 \pm 0.07$ & $-156 \pm 20$ & $0.114 \pm 0.011$ & $\mathrm{C} 2$ \\
\hline & $1.83 \pm 0.18$ & $0.84 \pm 0.08$ & $-152 \pm 15$ & $0.164 \pm 0.016$ & $\mathrm{C} 3$ \\
\hline & $0.90 \pm 0.13$ & $1.03 \pm 0.10$ & $-137 \pm 22$ & $0.753 \pm 0.075$ & $\mathrm{X}$ \\
\hline 1643 & $8.14 \pm 0.81$ & $0 \pm 0$ & $0 \pm 0$ & $0.062 \pm 0.006$ & core \\
\hline \multirow[t]{5}{*}{ (2013 Dec) } & $1.66 \pm 0.16$ & $0.25 \pm 0.02$ & $-166 \pm 16$ & $0.165 \pm 0.016$ & $\mathrm{NC} 1$ \\
\hline & $3.52 \pm 0.35$ & $0.62 \pm 0.06$ & $-156 \pm 15$ & $0.136 \pm 0.013$ & $\mathrm{C} 1$ \\
\hline & $2.46 \pm 0.24$ & $0.78 \pm 0.07$ & $-154 \pm 18$ & $0.078 \pm 0.007$ & $\mathrm{C} 2$ \\
\hline & $1.02 \pm 0.10$ & $0.87 \pm 0.08$ & $-150 \pm 15$ & $0.134 \pm 0.013$ & $\mathrm{C} 3$ \\
\hline & $0.55 \pm 0.08$ & $1.08 \pm 0.10$ & $-132 \pm 11$ & $0.653 \pm 0.065$ & $\mathrm{X}$ \\
\hline 1678 & $6.77 \pm 0.67$ & $0 \pm 0$ & $0 \pm 0$ & $0.077 \pm 0.007$ & core \\
\hline \multirow[t]{5}{*}{ (2014 Jan) } & $2.52 \pm 0.25$ & $0.24 \pm 0.02$ & $-165 \pm 16$ & $0.172 \pm 0.017$ & $\mathrm{NC} 1$ \\
\hline & $1.60 \pm 0.16$ & $0.53 \pm 0.05$ & $-158 \pm 15$ & $0.083 \pm 0.008$ & $\mathrm{X}$ \\
\hline & $3.48 \pm 0.34$ & $0.72 \pm 0.07$ & $-155 \pm 15$ & $0.094 \pm 0.009$ & $\mathrm{C} 1$ \\
\hline & $2.25 \pm 0.22$ & $0.87 \pm 0.08$ & $-152 \pm 15$ & $0.165 \pm 0.016$ & $\mathrm{C} 2$ \\
\hline & $0.87 \pm 0.08$ & $1.03 \pm 0.10$ & $-137 \pm 13$ & $0.672 \pm 0.067$ & $\mathrm{C} 3$ \\
\hline 1714 & $6.98 \pm 0.69$ & $0 \pm 0$ & $0 \pm 0$ & $0.082 \pm 0.008$ & core \\
\hline \multirow[t]{5}{*}{ (2014 Feb) } & $2.59 \pm 0.25$ & $0.36 \pm 0.03$ & $-162 \pm 16$ & $0.164 \pm 0.016$ & $\mathrm{NC} 1$ \\
\hline & $1.65 \pm 0.16$ & $0.60 \pm 0.06$ & $-157 \pm 15$ & $0.085 \pm 0.008$ & $\mathrm{X}$ \\
\hline & $3.36 \pm 0.33$ & $0.76 \pm 0.07$ & $-154 \pm 15$ & $0.087 \pm 0.008$ & $\mathrm{C} 1$ \\
\hline & $1.95 \pm 0.19$ & $0.88 \pm 0.08$ & $-151 \pm 15$ & $0.164 \pm 0.016$ & $\mathrm{C} 2$ \\
\hline & $0.61 \pm 0.06$ & $1.13 \pm 0.11$ & $-133 \pm 13$ & $0.775 \pm 0.077$ & $\mathrm{C} 3$ \\
\hline 1781 & $6.51 \pm 0.65$ & $0 \pm 0$ & $0 \pm 0$ & $0.073 \pm 0.007$ & core \\
\hline \multirow[t]{5}{*}{ (2014 May) } & $2.20 \pm 0.22$ & $0.25 \pm 0.02$ & $-172 \pm 17$ & $0.123 \pm 0.012$ & $\mathrm{NC} 2$ \\
\hline & $2.22 \pm 0.22$ & $0.49 \pm 0.04$ & $-161 \pm 16$ & $0.096 \pm 0.009$ & $\mathrm{NC} 1$ \\
\hline & $2.67 \pm 0.26$ & $0.79 \pm 0.07$ & $-154 \pm 15$ & $0.115 \pm 0.011$ & $\mathrm{C} 1$ \\
\hline & $3.20 \pm 0.32$ & $0.94 \pm 0.09$ & $-151 \pm 15$ & $0.243 \pm 0.024$ & $\mathrm{C} 2$ \\
\hline & $0.24 \pm 0.02$ & $1.73 \pm 0.17$ & $-148 \pm 14$ & $0.242 \pm 0.024$ & $\mathrm{X}$ \\
\hline 1829 & $5.12 \pm 0.51$ & $0 \pm 0$ & $0 \pm 0$ & $0.068 \pm 0.006$ & core \\
\hline \multirow[t]{5}{*}{ (2014 Jun) } & $2.47 \pm 0.24$ & $0.28 \pm 0.02$ & $-161 \pm 16$ & $0.078 \pm 0.007$ & $\mathrm{NC} 2$ \\
\hline & $2.21 \pm 0.22$ & $0.52 \pm 0.05$ & $-162 \pm 16$ & $0.065 \pm 0.006$ & $\mathrm{NC} 1$ \\
\hline & $2.03 \pm 0.20$ & $0.77 \pm 0.07$ & $-153 \pm 15$ & $0.144 \pm 0.014$ & $\mathrm{C} 1$ \\
\hline & $1.51 \pm 0.15$ & $1.01 \pm 0.10$ & $-151 \pm 15$ & $0.176 \pm 0.017$ & $\mathrm{C} 2$ \\
\hline & $0.60 \pm 0.06$ & $1.27 \pm 0.12$ & $-135 \pm 13$ & $0.716 \pm 0.071$ & $\mathrm{C} 3$ \\
\hline 1867 & $5.71 \pm 0.57$ & $0 \pm 0$ & $0 \pm 0$ & $0.076 \pm 0.007$ & core \\
\hline \multirow[t]{6}{*}{ (2014 Jul) } & $2.09 \pm 0.20$ & $0.29 \pm 0.02$ & $-165 \pm 16$ & $0.233 \pm 0.023$ & $\mathrm{NC} 2$ \\
\hline & $2.11 \pm 0.21$ & $0.50 \pm 0.05$ & $-162 \pm 16$ & $0.046 \pm 0.004$ & $\mathrm{X}$ \\
\hline & $1.86 \pm 0.23$ & $0.68 \pm 0.06$ & $-156 \pm 15$ & $0.108 \pm 0.010$ & $\mathrm{NC} 1$ \\
\hline & $1.51 \pm 0.15$ & $0.83 \pm 0.08$ & $-149 \pm 14$ & $0.114 \pm 0.011$ & $\mathrm{C} 1$ \\
\hline & $1.25 \pm 0.15$ & $1.13 \pm 0.11$ & $-152 \pm 15$ & $0.257 \pm 0.025$ & $\mathrm{C} 2$ \\
\hline & $0.42 \pm 0.04$ & $1.43 \pm 0.14$ & $-129 \pm 12$ & $0.733 \pm 0.073$ & $\mathrm{C} 3$ \\
\hline 1924 & $5.80 \pm 0.58$ & $0 \pm 0$ & $0 \pm 0$ & $0.056 \pm 0.005$ & core \\
\hline \multirow[t]{6}{*}{ (2014 Sep) } & $1.89 \pm 0.18$ & $0.25 \pm 0.02$ & $-157 \pm 15$ & $0.137 \pm 0.013$ & $\mathrm{X}$ \\
\hline & $1.78 \pm 0.17$ & $0.52 \pm 0.05$ & $-159 \pm 19$ & $0.048 \pm 0.004$ & $\mathrm{NC} 2$ \\
\hline & $2.44 \pm 0.24$ & $0.74 \pm 0.09$ & $-156 \pm 15$ & $0.098 \pm 0.009$ & $\mathrm{NC} 1$ \\
\hline & $1.65 \pm 0.16$ & $0.87 \pm 0.08$ & $-148 \pm 18$ & $0.176 \pm 0.017$ & $\mathrm{C} 1$ \\
\hline & $0.73 \pm 0.07$ & $1.20 \pm 0.12$ & $-151 \pm 15$ & $0.198 \pm 0.019$ & $\mathrm{C} 2$ \\
\hline & $0.47 \pm 0.04$ & $1.52 \pm 0.15$ & $-130 \pm 20$ & $0.713 \pm 0.071$ & $\mathrm{C} 3$ \\
\hline 1976 & $4.59 \pm 0.45$ & $0 \pm 0$ & $0 \pm 0$ & $0.059 \pm 0.005$ & core \\
\hline \multirow[t]{5}{*}{ (2014 Nov) } & $0.83 \pm 0.08$ & $0.23 \pm 0.02$ & $-159 \pm 15$ & $0.107 \pm 0.010$ & $\mathrm{X}$ \\
\hline & $1.10 \pm 0.11$ & $0.53 \pm 0.05$ & $-159 \pm 19$ & $0.066 \pm 0.006$ & $\mathrm{NC} 2$ \\
\hline & $2.45 \pm 0.56$ & $0.79 \pm 0.07$ & $-154 \pm 15$ & $0.147 \pm 0.014$ & $\mathrm{NC} 1$ \\
\hline & $0.69 \pm 0.06$ & $1.06 \pm 0.10$ & $-149 \pm 14$ & $0.258 \pm 0.025$ & $\mathrm{C} 1$ \\
\hline & $0.51 \pm 0.05$ & $1.54 \pm 0.15$ & $-134 \pm 17$ & $0.935 \pm 0.093$ & $\mathrm{C} 3$ \\
\hline 1997 & $5.90 \pm 0.59$ & $0 \pm 0$ & $0 \pm 0$ & $0.068 \pm 0.006$ & core \\
\hline
\end{tabular}


Table 2

(Continued)

\begin{tabular}{|c|c|c|c|c|c|}
\hline $\begin{array}{l}\text { Epoch }\left(\mathrm{JD}^{\prime}\right) \\
\text { Date }\end{array}$ & $\begin{array}{c}S \\
(\mathrm{Jy})\end{array}$ & $\begin{array}{c}r \\
\text { (mas) }\end{array}$ & $\begin{array}{c}\theta \\
(\mathrm{deg})\end{array}$ & $\begin{array}{c}\phi \\
\text { (mas) }\end{array}$ & Comp $^{\mathrm{a}}$ \\
\hline (2014 Dec) & $\begin{array}{l}1.33 \pm 0.13 \\
0.72 \pm 0.07 \\
0.95 \pm 0.09 \\
2.31 \pm 0.23 \\
0.53 \pm 0.05 \\
0.27 \pm 0.05 \\
0.32 \pm 0.03 \\
0.08 \pm 0.02\end{array}$ & $\begin{array}{l}0.16 \pm 0.01 \\
0.48 \pm 0.04 \\
0.68 \pm 0.06 \\
0.84 \pm 0.08 \\
0.97 \pm 0.09 \\
1.28 \pm 0.11 \\
1.61 \pm 0.14 \\
1.65 \pm 0.19\end{array}$ & $\begin{array}{l}-162 \pm 16 \\
-160 \pm 16 \\
-158 \pm 15 \\
-154 \pm 15 \\
-147 \pm 19 \\
-150 \pm 15 \\
-127 \pm 19 \\
-153 \pm 15\end{array}$ & $\begin{array}{l}0.115 \pm 0.011 \\
0.050 \pm 0.009 \\
0.108 \pm 0.010 \\
0.148 \pm 0.014 \\
0.198 \pm 0.019 \\
0.236 \pm 0.023 \\
0.667 \pm 0.066 \\
0.325 \pm 0.032\end{array}$ & $\begin{array}{c}\mathrm{NC} 3 \\
\mathrm{X} \\
\mathrm{NC} 2 \\
\mathrm{NC} 1 \\
\mathrm{C} 1 \\
\mathrm{C} 2 \\
\mathrm{C} 3 \\
\mathrm{X}\end{array}$ \\
\hline
\end{tabular}

Note. $\mathrm{JD}^{\prime}=\mathrm{JD}-2,454,000 ; S$ : integrated flux density in the component; $r$ : radial distance of the component center from the center of the map; $\theta$ : position angle of the center of the component; $\phi$ : FWHM of the component; Comp $p^{\mathrm{a}}$ : identification of the individual components. If a component appeared only in a single epoch, we labeled it with X.

\section{ORCID iDs}

S. G. Jorstad (10) https://orcid.org/0000-0001-6158-1708

A. P. Marscher (iD https://orcid.org/0000-0001-7396-3332

V. M. Larionov (iD https://orcid.org/0000-0002-4640-4356

\section{References}

Abdo, A. A., Ackermann, M., Agudo, I., et al. 2010, ApJ, 716, 30 Acero, F., Ackermann, M., Ajello, M., et al. 2015, ApJS, 218, 23 Ackermann, M., Anantua, R., Asano, K., et al. 2016, ApJL, 824, L20 Agudo, I., Marscher, A. P., Jorstad, S. G., et al. 2011, ApJL, 735, L10 Agudo, I., Thum, C., Gómez, J. L., \& Wiesemeyer, H. 2014, A\&A, 566, A59 Agudo, I., Thum, C., Molina, S. N., et al. 2018a, MNRAS, 474, 1427 Agudo, I., Thum, C., Ramakrishnan, V., et al. 2018b, MNRAS, 473, 1850 Agudo, I., Thum, C., Wiesemeyer, H., \& Krichbaum, T. P. 2010, ApJS, 189, 1 Albert, J., Aliu, E., Anderhub, H., et al. 2008, Sci, 320, 1752

Atwood, W. B., Abdo, A. A., Ackermann, M., et al. 2009, ApJ, 697, 1071

Blinov, D. A., \& Hagen-Thorn, V. A. 2009, A\&A, 503, 103

Bonning, E., Urry, C. M., Bailyn, C., et al. 2012, ApJ, 756, 13

Böttcher, M., Basu, S., Joshi, M., et al. 2007, ApJ, 670, 968

Burbidge, E. M., \& Rosenberg, F. D. 1965, ApJ, 142, 1673

Burrows, D. N., Hill, J. E., Nousek, J. A., et al. 2005, SSRv, 120, 165

Chatterjee, R., Jorstad, S. G., Marscher, A. P., et al. 2008, ApJ, 689, 79

Cheung, C. C. 2002, ApJL, 581, L15

Chidiac, C., Rani, B., Krichbaum, T. P., et al. 2016, A\&A, 590, A61

Collmar, W., Böttcher, M., Krichbaum, T. P., et al. 2010, A\&A, 522, A66

Connolly, S. 2015, arXiv:1503.06676

Cutini, S. 2015, ATel, 7633, 1

Emmanoulopoulos, D., McHardy, I. M., \& Papadakis, I. E. 2013, MNRAS, 433, 907

Fromm, C. M., Perucho, M., Ros, E., et al. 2012, IJMPS, 8, 323

Gómez, J. L., Martí, J. M., Marscher, A. P., Ibáñez, J. M., \& Alberdi, A. 1997, ApJL, 482, L33

Gu, M., Cao, X., \& Jiang, D. R. 2001, MNRAS, 327, 1111

Gurwell, M. A., Peck, A. B., Hostler, S. R., Darrah, M. R., \& Katz, C. A. 2007, in ASP Conf. Ser. 375, From Z-Machines to ALMA: (Sub)Millimeter Spectroscopy of Galaxies, ed. A. J. Baker et al. (San Francisco, CA: ASP), 234

Hartman, R. C., Bertsch, D. L., Fichtel, C. E., et al. 1992, ApJL, 385, L1

Hartman, R. C., Webb, J. R., Marscher, A. P., et al. 1996, ApJ, 461, 698

Hayashida, M., Madejski, G. M., Nalewajko, K., et al. 2012, ApJ, 754, 114

Hayashida, M., Nalewajko, K., Madejski, G. M., et al. 2015, ApJ, 807, 79

Hodgson, J. A., Krichbaum, T. P., Marscher, A. P., et al. 2017, A\&A, 597, A80

Homan, D. C., Lister, M. L., Aller, H. D., Aller, M. F., \& Wardle, J. F. C. 2009, ApJ, 696, 328

Jorstad, S., \& Marscher, A. 2016, Galax, 4, 47
Jorstad, S. G., Marscher, A. P., Larionov, V. M., et al. 2010, ApJ, 715, 362 Jorstad, S. G., Marscher, A. P., Lister, M. L., et al. 2004, AJ, 127, 3115 Jorstad, S. G., Marscher, A. P., Lister, M. L., et al. 2005, AJ, 130, 1418 Jorstad, S. G., Marscher, A. P., Morozova, D. A., et al. 2017, ApJ, 846, 98 Kalberla, P. M. W., Burton, W. B., Hartmann, D., et al. 2005, A\&A, 440, 775 Kang, S., Lee, S.-S., \& Byun, D.-Y. 2015, JKAS, 48, 257

Katarzyński, K., \& Ghisellini, G. 2007, A\&A, 463, 529

Larionov, V. M., Jorstad, S. G., Marscher, A. P., et al. 2008, A\&A, 492, 389

Lisakov, M. M., Kovalev, Y. Y., Savolainen, T., Hovatta, T., \& Kutkin, A. M. 2017, MNRAS, 468, 4478

Lister, M. L., Aller, M. F., Aller, H. D., et al. 2013, AJ, 146, 120

Lott, B., Escande, L., Larsson, S., \& Ballet, J. 2012, A\&A, 544, A6

Maraschi, L., Grandi, P., Urry, C. M., et al. 1994, ApJL, 435, L91

Marscher, A. P. 2008, in ASP Conf. Ser. 386, Extragalactic Jets: Theory and Observation from Radio to Gamma Ray, ed. T. A. Rector \& D. S. De Young (San Francisco, CA: ASP), 437

Marscher, A. P. 2014, ApJ, 780, 87

Mattox, J. R., Bertsch, D. L., Chiang, J., et al. 1996, ApJ, 461, 396

Mead, A. R. G., Ballard, K. R., Brand, P. W. J. L., et al. 1990, A\&AS, 83, 183

Meyer, E. T., Georganopoulos, M., Sparks, W. B., et al. 2015, Natur, 521, 495

Nalewajko, K., \& Begelman, M. C. 2012, MNRAS, 427, 2480

Nishikawa, K.-I., Mizuno, Y., Niemiec, J., et al. 2016, Galax, 4, 38

Poole, T. S., Breeveld, A. A., Page, M. J., et al. 2008, MNRAS, 383, 627

Rani, B., Krichbaum, T. P., Hodgson, J. A., \& Zensus, J. A. 2016, J. Phys. Conf. Ser., 718, 052032

Rani, B., Krichbaum, T. P., Lee, S.-S., et al. 2017, MNRAS, 464, 418

Rani, B., Krichbaum, T. P., Marscher, A. P., et al. 2014, A\&A, 571, L2

Rani, B., Krichbaum, T. P., Marscher, A. P., et al. 2015, A\&A, 578, A123

Richards, J. L., Max-Moerbeck, W., Pavlidou, V., et al. 2011, ApJS, 194, 29

Roming, P. W. A., Kennedy, T. E., Mason, K. O., et al. 2005, SSRv, 120, 95

Schlegel, D. J., Finkbeiner, D. P., \& Davis, M. 1998, ApJ, 500, 525

Smith, P. 2016, Galax, 4, 27

Shepherd, M. C., Pearson, T. J., \& Taylor, G. B. 1994, BAAS, 26, 987

Spergel, D. N., Verde, L., Peiris, H. V., et al. 2003, ApJS, 148, 175

Taylor, G. B. 2000, ApJ, 533, 95

Thum, C., Agudo, I., Molina, S. N., et al. 2018, MNRAS, 473, 2506

Thum, C., Wiesemeyer, H., Paubert, G., Navarro, S., \& Morris, D. 2008, PASP, 120, 777

Vaughan, S., Edelson, R., Warwick, R. S., \& Uttley, P. 2003, MNRAS, 345,1271

Wagner, S. J., \& Mannheim, K. 2001, in ASP Conf. Ser. 250, Particles and Fields in Radio Galaxies Conference, ed. R. A. Laing \& K. M. Blundell (San Francisco, CA: ASP), 142

Wardle, J. F. C., Homan, D. C., Ojha, R., \& Roberts, D. H. 1998, Natur, 395,457

Wehrle, A. E., Pian, E., Urry, C. M., et al. 1998, ApJ, 497, 178

Woo, J.-H., \& Urry, C. M. 2002, ApJ, 579, 530 